\title{
Review \\ Ethnobotany, Phytochemistry, Biological, and Nutritional Properties of Genus Crepis-A Review
}

\author{
Natale Badalamenti ${ }^{1}\left(\mathbb{D}\right.$, Francesco Sottile $^{2,3}$ and Maurizio Bruno and $^{1,3, * \mathbb{C}}$ \\ 1 Department of Biological, Chemical and Pharmaceutical Sciences and Technologies (STEBICEF), \\ Università degli Studi di Palermo, Viale delle Scienze, Ed. 17, I-90128 Palermo, Italy; \\ natale.badalamenti@unipa.it \\ 2 Department of Architecture, University of Palermo, Viale delle Scienze, Parco d'Orleans II, \\ I-90128 Palermo, Italy; francesco.sottile@unipa.it \\ 3 Centro Interdipartimentale di Ricerca "Riuszo Bio-Based Degli Scarti da Matrici Agroalimentari" (RIVIVE), \\ Università degli Studi di Palermo, I-90128 Palermo, Italy \\ * Correspondence: maurizio.bruno@unipa.it
}

Citation: Badalamenti, N.; Sottile, F.; Bruno, M. Ethnobotany,

Phytochemistry, Biological, and Nutritional Properties of Genus Crepis-A Review. Plants 2022, 11, 519. https://doi.org/10.3390/ plants11040519

Academic Editors: Luisa Pistelli and Basma Najar

Received: 26 January 2022

Accepted: 11 February 2022

Published: 14 February 2022

Publisher's Note: MDPI stays neutral with regard to jurisdictional claims in published maps and institutional affiliations.

Copyright: (C) 2022 by the authors. Licensee MDPI, Basel, Switzerland. This article is an open access article distributed under the terms and conditions of the Creative Commons Attribution (CC BY) license (https:// creativecommons.org/licenses/by/ $4.0 /)$.

\begin{abstract}
The genus Crepis L., included within the Asteraceae family, has a very wide distribution, expanding throughout the northern hemisphere, including Europe, northern Africa, and temperate Asia. This genus has a fundamental value from biodynamic and ecological perspectives, with the different species often being chosen for soil conservation, for environmental sustainability, and for their attraction towards pollinating species. Furthermore, various species of Crepis have been used in the popular medicine of several countries as medicinal herbs and food since ancient times. In most cases, the species is consumed either in the form of a decoction, or as a salad, and is used for its cardiovascular properties, as a digestive, for problems related to sight, for the treatment of diabetes, and for joint diseases. This literature review, the first one of the Crepis genus, includes publications with the word 'Crepis', and considers the single metabolites identified, characterised, and tested to evaluate their biological potential. The various isolated compounds, including in most cases sesquiterpenes and flavonoids, were obtained by extracting the roots and aerial parts of the different species. The secondary metabolites, extracted using traditional (solvent extraction, column chromatography, preparative thin layer chromatography, preparative HPLC, vacuum liquid chromatography), and modern systems such as ultrasounds, microwaves, etc., and characterised by mono- and bi- dimensional NMR experiments and by HPLC-MS, have a varied application spectrum at a biological level, with antimicrobial, antioxidant, antidiabetic, antitumor, antiviral, antiulcer, phytotoxic, and nutritional properties having been reported. Unfortunately, in vitro tests have not always been accompanied by in vivo tests, and this is the major critical aspect that emerges from the study of the scientific aspects related to this genus. Therefore, extensive investigations are necessary to evaluate the real capacity of the different species used in food, and above all to discover what the different plants that have never been analysed could offer at a scientific level.
\end{abstract}

Keywords: Crepis ssp.; Asteraceae; secondary metabolites; sesquiterpenes; Guiaianes; ethnopharmacology; biological properties

\section{Introduction}

The genus Crepis L. belongs to the tribe Cichorieae Lam. \& DC (the old name is Lactuceae Cass.) of the Asteraceae family. This tribe is characterised by ligulate florets, which are commonly five lobed, and by milky juice, and it includes more than 98 genera and 1550 species [1] (Figure 1). Under the tribe Cichorieae, there are fourteen sub-tribes, one of which is Crepidinae, which was re-recognised by Kilian et al. [1] with 22 genera (including Crepis). The genus Crepis L. (hawk's beard) is the second largest genus in the tribe, with more than 200 species [2]. Actually, The Plant List [3] shows 1302 plant name records, of which 293 rank as accepted species or sub-species. Species of this genus grow in 
the northern hemisphere, with just a few being present in Southeast Asia. Some species also occur in East, South, and West Africa, in the Canary Islands, and Madeira. According to Babcock [4], Crepis originated in the Altai/Tien Shan region in Central Asia, although, at present, the centre of diversification is the circum-Mediterranean area. Crepis species can grow in different types of habitats ranging from alpine zone, swamps, low grasslands, and forests to beaches. Size ranges from only a few centimetres in height (e.g., C. pygmaea) to nearly two metres in C. sibirica [5].
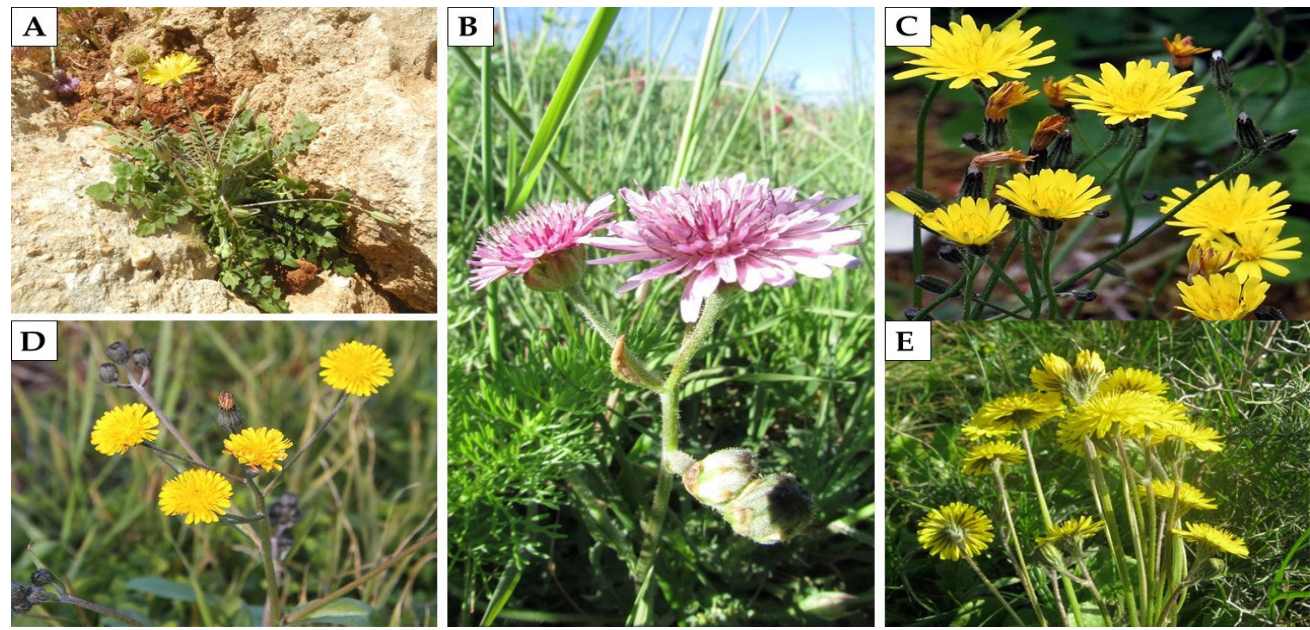

Figure 1. Some of the Crepis ssp. present in Sicily, Italy: (A) C. bursifolia L., (B) C. bivoniana (Rchb.) Soldano \& F. Conti, (C) C. leontodontoides All., (D) C. vesicaria L., and (E) C. sancta (L.) Bornm.

Crepis L. has significant value from an agronomic and ecosystem perspective, mostly in Mediterranean environments. The role of wild weed mixtures in fields has always been complex, and now takes on renewed importance, especially today, when agriculture plays a not insignificant role in biodiversity loss [6,7]. Industrialised agricultural models, increasingly dependent on the use of synthetic chemicals (fertilisers, pesticides, and herbicides) have resulted in strong selection in wild flora with a substantial reduction in ecosystem services [8]. This specialisation is not a positive factor for either beneficial insect biodiversity [9] or improved soil characteristics and fertility preservation.

Crepis L. species have often been found in organically cultivated fields [10,11], i.e., fields that have not undergone invasive soil actions in which floristic communities play a key role in soil conservation and increasing ecosystem biodiversity [12]. Specifically, Crepis sancta is often present in natural cover crops of vineyards in France [13], contributing to the creation of planting systems with high environmental sustainability. Moreover, the presence of Crepis L. in wild grass mixtures appears to be significantly influenced by the fallow that preceded plantings [14]. In other environments, C. biennis, C. vesicaria, and C. capillaris have been used in seed mixtures to establish ecological corridors for pollinators $[15,16]$. C. biennis, moreover, has been used with positive effects in seed mixtures for vegetative cover of fallow soils in urban environments [17].

Consequently, due to our expertise in the Asteraceae family [18-24], and to the absence of any literature review on Crepis genus, we decided to analyse and survey all papers concerning this genus.

\section{Review Methodology}

In this review, a complete survey of the chemical composition, and biological properties of the essential oils, extracts, and non-volatile compounds isolated from Crepis taxa is provided. Moreover, the agronomical and traditional uses of Crepis ssp. are also reported.

The search terms included the word Crepis, all the botanical names of the species, both accepted names or synonyms, using the electronic databases PubMed, SciFinder, Science Direct, Scopus, Web of Science, and Google Scholar. We used the principles of PRISMA to 
conduct this review (Figure 2) [25]. The available information on these genera was collected from scientific databases, and cover from 1911 up to 2021. Essential oils, metabolites, traditional uses, biological activity, and toxicity are the topics of this literature review. No limitations were set for languages. Table S1 reports the taxa of Crepis investigated so far with their botanical authority, their synonyms, and the accepted botanical names, according to The Plant List [3].

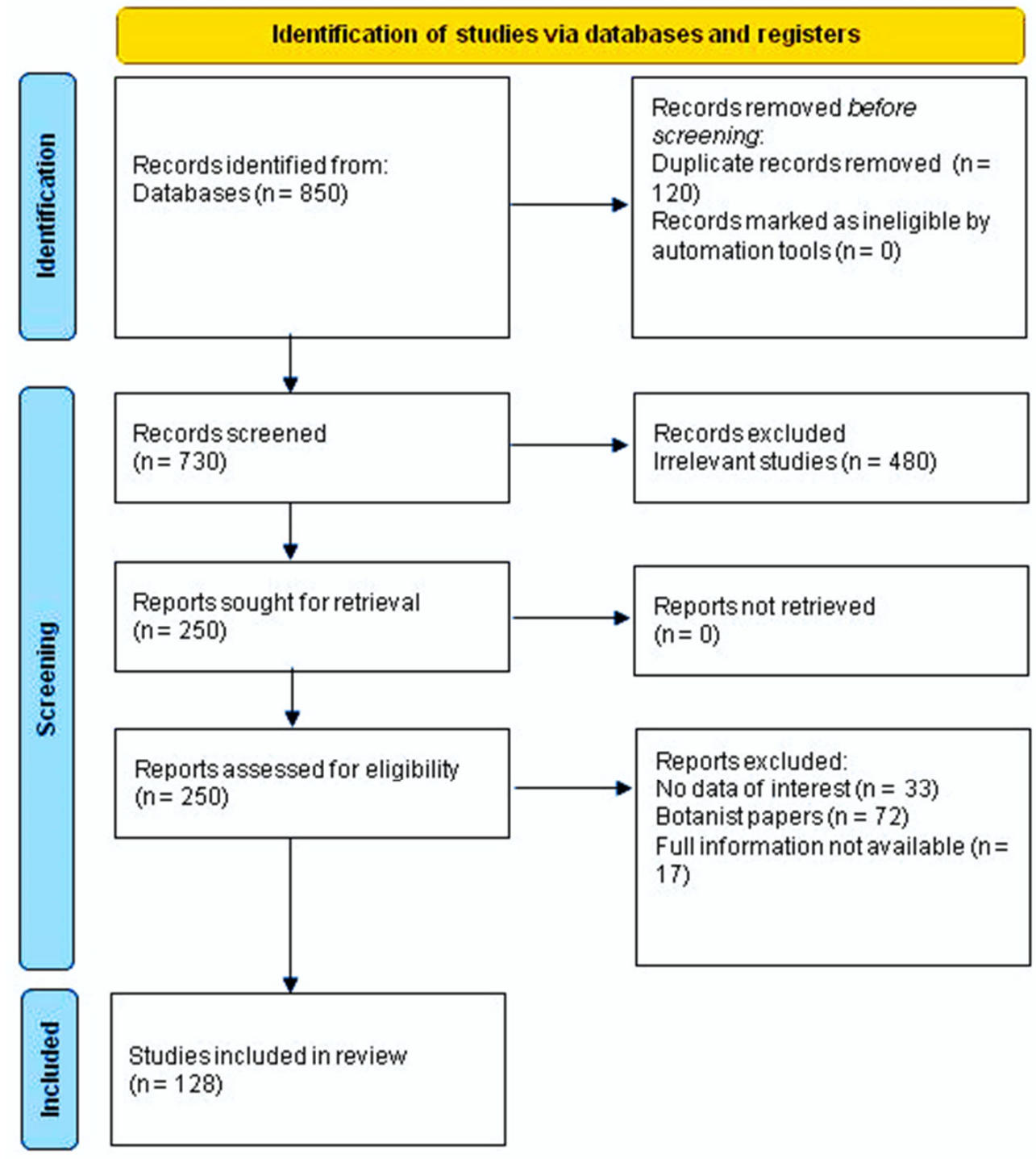

Figure 2. PRISMA 2020 flow diagram for new systematic reviews, which includes database and register searches only.

In the present review, all the species have been reported as quoted in the original works although some of them are synonyms (Table S1).

\section{Alimentary Properties and Traditional Uses}

\subsection{Traditional Uses}

Crepis species have been used as medicinal herbs and food for several centuries, and many of them are also currently in use in folk medicine. A summary of their traditional uses is presented in Table 1. 
Table 1. Ethnobotanical use of Crepis taxa.

\begin{tabular}{|c|c|c|c|c|c|}
\hline Taxa & Area & Parts & Vernacular Name & Properties & Ref. \\
\hline C. biennis & Abruzzo, Italy & young leaves & cicorietta selvatica & $\begin{array}{l}\text { Ingredients of the soup 'brodo del } \\
\text { pastore'. Eaten in salads }\end{array}$ & [26] \\
\hline C. bivoniana & Sicily, Italy & leaves & luciazzi & Used in soups, salads and omelettes & [27] \\
\hline \multirow[t]{2}{*}{ C. bursifolia } & Campania, Italy & aerial parts & margarita & $\begin{array}{l}\text { Aerial part used in the preparation of } \\
\text { 'minestra terrana'. Basal leaves in } \\
\text { salads and soups }\end{array}$ & [28] \\
\hline & Sicily, Italy & leaves & $\begin{array}{l}\text { ricuttedda; } \\
\text { rizzaredda }\end{array}$ & $\begin{array}{l}\text { Boiled or pan fried leaves as food. } \\
\text { Diuretic and antioxidant }\end{array}$ & {$[27,29]$} \\
\hline C. cameroonica & Cameroon & & & $\begin{array}{l}\text { Treatment of diarrhoea, wounds and } \\
\text { fungal infections }\end{array}$ & {$[30]$} \\
\hline C. capillaris & Tuscany, Italy & leaves & & $\begin{array}{c}\text { Raw or boiled leaves are used for } \\
\text { salads and soups. } \\
\text { Laxative properties. }\end{array}$ & {$[31]$} \\
\hline C. carbonaria & Africa & & & Myometrial contractions & [32] \\
\hline C. commutata & Crete, Greece & leaves & glucosides & $\begin{array}{l}\text { Leaves are usually eaten boiled } \\
\text { in salads }\end{array}$ & {$[33,34]$} \\
\hline C. crocea & China & & & $\begin{array}{l}\text { Relieving cough and asthma, } \\
\text { antipyretic, benefitting Qi, and } \\
\text { reducing inflammation }\end{array}$ & [35] \\
\hline C. flexuosa & Himalaya & whole plant & homa-sili & For jaundice and liver disorder & {$[36,37]$} \\
\hline \multirow{3}{*}{$\begin{array}{l}\text { C. foetida ssp. } \\
\text { rhoeadifolia }\end{array}$} & Turkey & aerial parts & yürek out & For cardiovascular diseases & {$[38,39]$} \\
\hline & Turkey & leaves & kohum & Food & {$[40]$} \\
\hline & & & & $\begin{array}{l}\text { Anti-cancerous and wound } \\
\text { healing agent }\end{array}$ & [41] \\
\hline C. glauca & Utah, USA & leaves & & $\begin{array}{l}\text { Leaves have been eaten among } \\
\text { Gosiute Indians }\end{array}$ & {$[42]$} \\
\hline C. hakkarica & Turkey & aerial parts & tahliş & $\begin{array}{l}\text { A decoction of the leaves and flowers } \\
\text { is used in the treatment of diabetes }\end{array}$ & [43] \\
\hline C. hyemalis & Sicily, Italy & leaves & luciazzi & Used in soups, salads and with eggs & \\
\hline \multirow[t]{2}{*}{ C. japonica } & China & & & $\begin{array}{l}\text { For reducing pyrexia, detoxification } \\
\text { and atopy. Antitussive, febrifuge. It is } \\
\text { also used in the treatment of boils } \\
\text { and snakebites. Internally for the } \\
\text { remedies of cold, sore throat, and } \\
\text { diarrhoea, and externally as } \\
\text { medicated paste to relieve shingles }\end{array}$ & [44,45] \\
\hline & Bangladesh & leaves $\mathrm{r}$. & crepis & $\begin{array}{l}\text { The leaves applied to wounds act as a } \\
\text { styptic and heal them quickly. The } \\
\text { juice of the root possesses } \\
\text { antilithic properties. }\end{array}$ & [46] \\
\hline \multirow[t]{2}{*}{ C. lacera } & Italy & & & $\begin{array}{l}\text { Fatal if frequently ingested by } \\
\text { ruminants such as sheep and cattle. } \\
\text { Detoxification, purification, diuretic } \\
\text { and hypoglycemic effects }\end{array}$ & {$[47]$} \\
\hline & Campania, Italy & aerial parts & & $\begin{array}{l}\text { Young basal leaves, flower buds in } \\
\text { salads or cooked vegetables }\end{array}$ & [28] \\
\hline
\end{tabular}


Table 1. Cont.

\begin{tabular}{|c|c|c|c|c|c|}
\hline Taxa & Area & Parts & Vernacular Name & Properties & Ref. \\
\hline \multirow{2}{*}{ C. leontodontoides } & Campania, Italy & aerial parts & & $\begin{array}{l}\text { Aerial part as ingredient of 'minestra } \\
\text { terrana'. Young basal leaves, flower } \\
\text { buds in salads or cooked vegetables }\end{array}$ & [28] \\
\hline & Tuscany, Italy & leaves & & $\begin{array}{l}\text { Boiled leaves for salads and soups. } \\
\text { Laxative properties }\end{array}$ & [31] \\
\hline \multirow[b]{2}{*}{ C. neglecta } & Tuscany, Italy & leaves & & Boiled leaves for salads and soups & [31] \\
\hline & Campania, Italy & aerial parts & spaccapreti & $\begin{array}{l}\text { Young basal leaves, flower buds In } \\
\text { salads or cooked vegetables. }\end{array}$ & [28] \\
\hline $\begin{array}{l}\text { C. neglecta ssp. } \\
\text { corymbosa }\end{array}$ & Campania, Italy & aerial parts & lattarulo & Basal leaves in soups & [28] \\
\hline C. pulchra & Campania, Italy & aerial parts & & $\begin{array}{l}\text { Young basal leaves, flower buds in } \\
\text { salads or cooked vegetable }\end{array}$ & [28] \\
\hline C. reuteriana & Syria & young leaves & souraga & $\begin{array}{l}\text { As salad for relieve joint diseases } \\
\text { pain and as appetiser }\end{array}$ & [48] \\
\hline \multirow[b]{2}{*}{ C. rueppellii } & Yemen & & & $\begin{array}{c}\text { For hepatic disorders (jaundice, } \\
\text { hepatitis and gallstones) }\end{array}$ & [49] \\
\hline & Ethiopia & roots & & $\begin{array}{c}\text { Fresh roots are crushed and orally } \\
\text { given with water to livestock } \\
\text { against Anthrax }\end{array}$ & [50] \\
\hline \multirow{5}{*}{ C. sancta } & Turkey & stems & düğmelik & Cooked as digestive & [51] \\
\hline & Turkey & fleaves & keklik otu & For eye diseases and as vasodilators & [52] \\
\hline & $\begin{array}{l}\text { Emilia Romagna } \\
\text { (Italy) }\end{array}$ & young leaves & ciocapiat & $\begin{array}{l}\text { Food, pan-fried or salad, and for its } \\
\text { diuretic and laxative effects }\end{array}$ & [53] \\
\hline & Tuscany, Italy & leaves & & $\begin{array}{l}\text { Boiled leaves are used for salads } \\
\text { and soups }\end{array}$ & [31] \\
\hline & Umbria, Italy & leaves & $\begin{array}{l}\text { crepide, dolcetta, } \\
\text { radicchiella di } \\
\text { terrasanta }\end{array}$ & $\begin{array}{c}\text { The young leaves are eaten, boiled } \\
\text { or raw }\end{array}$ & [54] \\
\hline \multirow[t]{2}{*}{ C. setosa } & Campania, Italy & aerial parts & $\begin{array}{l}\text { occhi neureddi; occhi } \\
\text { rossi; ragno purcello }\end{array}$ & $\begin{array}{l}\text { Young basal leaves, flower buds in } \\
\text { salads or cooked vegetable. Basal } \\
\text { leaves eaten in soups or as a } \\
\text { cooked vegetable }\end{array}$ & [28] \\
\hline & Tuscany, Italy & leaves & & $\begin{array}{l}\text { Boiled leaves are used for salads } \\
\text { and soups }\end{array}$ & [31] \\
\hline C. syriaca & Syria & young leaves & souraga & $\begin{array}{c}\text { As salad for relieve joint diseases } \\
\text { pain and as appetiser }\end{array}$ & [48] \\
\hline \multirow{5}{*}{ C. vesicaria } & Crete, Greece & leaves & & $\begin{array}{l}\text { Leaves are usually eaten boiled } \\
\text { in salads }\end{array}$ & [34] \\
\hline & Abruzzo, Italy & young leaves & $\begin{array}{l}\text { cicorietta selvatica; } \\
\text { cicorietta di } \\
\text { montagna }\end{array}$ & $\begin{array}{l}\text { Ingredients of soup "brodo del } \\
\text { pastore". Eaten in salads }\end{array}$ & [26] \\
\hline & Apulia, Italy & young leaves & cequariùnné & Cooked and consumed as vegetable & [55] \\
\hline & Campania, Italy & aerial parts & $\begin{array}{l}\text { lattarole; cicurioni; } \\
\text { scazzuoppoli; occhi } \\
\text { rossi }\end{array}$ & $\begin{array}{l}\text { Young basal leaves, flower buds in } \\
\text { salads or cooked vegetables. Aerial } \\
\text { part as ingredient of "minestra } \\
\text { maritata". Basal leaves eaten in soups } \\
\text { or as a cooked vegetable }\end{array}$ & [28] \\
\hline & Tuscany, Italy & leaves & & $\begin{array}{l}\text { Boiled leaves are used for salads } \\
\text { and soups }\end{array}$ & [31] \\
\hline
\end{tabular}


Table 1. Cont.

\begin{tabular}{|c|c|c|c|c|c|}
\hline Taxa & Area & Parts & Vernacular Name & Properties & Ref. \\
\hline & Umbria, Italy & leaves & $\begin{array}{l}\text { crepide; dolcetta; } \\
\text { radicchiella di } \\
\quad \text { terrasanta }\end{array}$ & $\begin{array}{c}\text { The young leaves are eaten, boiled } \\
\text { or raw }\end{array}$ & {$[54]$} \\
\hline & Lucania, Italy & leaves & $\begin{array}{l}\text { marolje; marosk; } \\
\text { liakra sperte }\end{array}$ & $\begin{array}{l}\text { Among the Arbëresh (ethnic } \\
\text { Albanian) communities the young } \\
\text { whorls are eaten boiled and fried }\end{array}$ & {$[56]$} \\
\hline & Sardinia, Italy & leaves & cicoria areste & $\begin{array}{l}\text { The broth of the boiled leaves is used } \\
\text { for abdominal colic. The raw or } \\
\text { cooked leaves have hypoglycemic, } \\
\text { laxative and hypertensive effects }\end{array}$ & {$[57,58]$} \\
\hline & Sicily, Italy & leaves & pizzocorvu & $\begin{array}{c}\text { Boiled leaves with laxative and } \\
\text { diuretic effects. Used in soups, salads } \\
\text { and with eggs. }\end{array}$ & {$[27,29]$} \\
\hline $\begin{array}{l}\text { C. vesicaria ssp. } \\
\text { haenseleri }\end{array}$ & Spain & aerial parts & arnica; flor de arnica & $\begin{array}{l}\text { For stomach ailments and problems } \\
\text { of arterial circulation; externally } \\
\text { applied for wound healing, bruises } \\
\text { and inflammations }\end{array}$ & [59] \\
\hline $\begin{array}{l}\text { C. vesicaria ssp. } \\
\text { taraxicifolia }\end{array}$ & $\begin{array}{l}\text { Emilia Romagna } \\
\text { (Italy) }\end{array}$ & young leaves & $\begin{array}{l}\text { radicchiella; } \\
\text { strecapugno }\end{array}$ & $\begin{array}{c}\text { Salads, pan-fried, omelettes, pasta } \\
\text { dough. Depurative, refreshing, blood } \\
\text { cleaning, diuretic, laxative (cooking } \\
\text { water, food) }\end{array}$ & {$[53]$} \\
\hline
\end{tabular}

Since ancient times, in Turkish folk medicine, the decoction (5\%) of the aerial parts of C. foetida L. subsp. rhoeadifolia (Bieb.) Celak, known as 'yürek out', is used for treatment of cardiovascular diseases [38,39]. Furthermore, its leaves, 'kohum', are consumed as food [40]. In the same area, the cooked stems of C. sancta (L.) Bornm. (düğmelik) are used as a digestive [51], whereas its raw flowers (keklik otu) are used for eye diseases and as vasodilators [52]. In the Van Province of Turkey, a decoction of the leaves and flowers of C. hakkarica Lamond, locally known as 'tahliş', is used in the treatment of diabetes [43]. The young leaves of both C. reuteriana Boiss., and C. syriaca (Bornm.) Babc. \& Navashin (souraga) are used in Syria as salad, to relieve joint diseases pain, and as an appetiser [48].

The leaves of C. japonica (L.) Benth (syn. Youngia japonica (L.) DC.) are widely used in the traditional Chinese medicine for reducing pyrexia, detoxification, and atopy. It is antitussive, febrifuge, and also used in the treatment of boils and snakebites. Internally, it is a remedy for cold, sore throat, and diarrhoea, and externally, it is applied as a medicated paste, and relieves shingles [44,45]. In Bangladesh, the leaves of the same species, applied to wounds, act as a styptic and heals them quickly. On the other hand, the juice of the root possesses antilithic properties [46]. In China, the cough- and asthma-relieving, antipyretic and inflammation-reduction properties of C. crocea (Lam.) Babcock have always been known [35].

In the Himalayan region of India, the fresh juice C. flexuosa (DC.), where it is known as 'Homa Sili', mixed with equal amount of water, is taken regularly once a day to cure jaundice until cured [36,37].

C. rueppellii Sch. Bip is used both in Asia and Africa, but with different purposes. In fact, in the popular medicine of Yemen it is used for hepatic disorders (jaundice, hepatitis, and gallstones) [49], whereas in Ethiopia, the fresh roots are crushed and given orally with water to livestock against Anthrax [50]. In Africa, there are ethnopharmacological reports for another two taxa, namely C. cameroonica Babcock ex Hutchinson \& Dalziel [syn. C. newii subsp. oliveriana (Kuntze) C.Jeffrey \& Beentje] and C. carbonaria Sch. Bip. The first one is used for the treatment of diarrhoea, wounds, and fungal infections [30]; the second one is used for myometrial contractions [32]. 
In Europe, the traditional use of species of genus Crepis is concentrated in the Mediterranean region. In Spain, an infusion of the aerial part with flowers of C. vesicaria L. subsp. haenseleri (Boiss.) P.D. Sell [syn. C. vesicaria subsp. taraxacifolia (Thuill.) Thell.] (vernacular names Arnica, Flor de arnica) is used internally for stomach upsets, and to improve the circulation. Externally, it is used with compresses and washes, or with poultices made from the boiled plant, and is used for inflammations, as an analgesic for bruises, as cicatrizant, and as an antiseptic [59]. On the island of Crete (Greece), the leaves of C. vesicaria L. and C. commutata (Spreng.) Greuter ( $\gamma \lambda \nu \kappa о \sigma \iota \rho i \delta \varepsilon \varsigma)$ are usually eaten boiled in salads $[33,34]$. As summarised in Table 1, a body of information has been reported on the use of Crepis taxa in Italy. The young leaves of C. sancta (L.) Bornm. are greatly appreciated in Emilia Romagna (Central Italy), where it is known as 'ciocapiat', as food, pan-fried or as salad, and for its diuretic and laxative effects [53]. They are also eaten, boiled or raw, in salads and soups in Tuscany and Umbria (Central Italy) [31,54]. Other species used as food in some parts of Italy and with therapeutic properties include C. bursifolia L. (diuretic and antoxidant, Sicily) [27,29], C. capillaris (L.) Wallr., C. leontodontoides All. (laxative, Tuscany) [31], C. lacera Ten. (diuretic and hypoglycemic) [47], C. vesicaria L. (hypoglycaemic, laxative and hypertensive, Sardinia) [57,58] and C. vesicaria L. subsp. taraxicifolia (Thuill) Thell. (diuretic and laxative, Emilia Romagna) [53].

\subsection{Nutritional Composition}

Spontaneous green leaf plants contain important quantities of essential macro- and micro-nutrients, and their content regulates the appearance of degenerative diseases, such as cancer, or cardiovascular diseases, etc. The nutritional content of wild species from the island of Crete, C. commutata and C. vesicaria, used in the culinary field as salads, was investigated in a study. The quantity of $\alpha$-tocopherol was evaluated $(0.36 \mathrm{mg} / 100 \mathrm{~g}$ for C. commutata, and $0.41 \mathrm{mg} / 100 \mathrm{~g}$ for C. vesicaria), which is a fat-soluble antioxidant that intervenes in the glutathione peroxidase pathway, protecting cell membranes from oxidation, and reacting with the lipid radicals produced in the chain reaction of lipid peroxidation, along with the total content of phenolic compounds (49.08 and $49.42 \mathrm{mg} / 100 \mathrm{~g}$, respectively), and the content of the main minerals such as $\mathrm{Na}, \mathrm{Ca}, \mathrm{Mg}, \mathrm{K}, \mathrm{Fe}$, and nitrites. The results suggested that a diet rich in these two plants provides the daily human needs with an excellent amount of vitamins, antioxidants, and minerals [60]. In turn, the nutritional content of the leaves of $C$. vesciaria subsp. taraxacifolia has been studied. It emerged that carbohydrates (with maltose being the most abundant of all) represented the most abundant macronutrient, followed by ash, proteins, and lipids. The lipid profile was moderate (0.69 g/100 g of fresh plant), with a predominance of polyunsaturated fatty acids, such as $\alpha$-linoleic acid. Additionally, in this case, the content of macro-minerals ( $\mathrm{Na}, \mathrm{Ca}, \mathrm{Fe}, \mathrm{K}, \mathrm{Mg}$, etc.) was moderate, corroborating the work performed by Simopoulos and Gopalan [60], and suggesting that the consumption of this plant as a supplementary food contributes to a balanced diet [61].

Finally, C. vesciaria L., normally consumed in the Mediterranean diet, has been tested for its nutritional composition and content of carotenoids, tocols, thiamine, and riboflavin. Small amounts of thiamine and riboflavin were found, but the plant was a source of xanthophylls (violaxanthin, neoxanthin, lutein, zeaxanthin and $\beta$-cryptoxanthin) and carotenes ( $\alpha$-carotene, $\beta$-carotene, 9-cis- $\beta$-carotene, and 13-cis- $\beta$-carotene). Lutein accounted for the highest content (about $4 \mathrm{mg} / 100 \mathrm{~g}$ ), but with good amounts of tocol, in particular $\alpha$ tocopherol (about 2-3 mg/100 g), suggesting it as a source of fibre, as well as vitamins A and $\mathrm{E}[62]$.

\section{Non-Volatile Metabolites}

\subsection{Occurrence}

In this section, the occurrence of secondary metabolites from roots and aerial parts of Crepis ssp. is investigated. The main class of compounds were sesquiterpenes, although several flavonoids, aromatics, and other mebolites were also identified. 
The identification of single isolated metabolites was conducted by spectroscopical methods as mass spectrometry (MS) infrared spectroscopy (IR), optical rotation, and 1Dand 2D-NMR. The analysis of several extracts was carried out by HPLC-MS. The analyses of oils were performed by GC-MS.

\subsection{Sesquiterpenes}

Differently from other tribes of the Asteraceae, which contain numerous metabolites with different sesquiterpene carbon skeletons, Cichorieae show the presence of only eudesmanes, germacranes, and guaianes. A common feature of sesquiterpenoids from the Cichorieae tribe is the presence of sugar or carboxylic acid residues in the molecules. Guaianolides are the most diversified group of sesquiterpenoids within the Cichorieae, and according to Zidorn [63], they can be divided in 13 different classes.

Phytochemical research on the thirty-one species of Crepis, studied so far for their sesquiterpenoidic content (Table 2), showed that those ones present in Crepis taxa belong to only three classes: the Costus lactone type, by far the most represented, with fifty-two compounds (1-52), hypocretenolides (53-55), and lactucin type (56-62) (Figures 3-9).

Table 2. Sesquiterpenes from Crepis ssp.

\begin{tabular}{|c|c|c|c|c|}
\hline Taxa & Origin & Parts & Sesquiterenes & Ref. \\
\hline C. aspera & Egypt & aerial parts & 8-epi-isolipidiol (42) & [64] \\
\hline \multirow[b]{2}{*}{ C. aurea } & Poland & roots & $\begin{array}{c}\text { 14-hydroxy-hypocretenolide (53), } \\
\text { 14-hydroxy-hypocretenolide- } \beta \text { - } D \text {-glucopyranoside (54), } \\
\text { 11,13 } \alpha \text {-dihydro-14-hydroxy-hypocretenolide- } \beta \text { - } D \text {-glucopyranoside (55) }\end{array}$ & [65] \\
\hline & Poland & roots & $\begin{array}{l}\text { 8-epi-deacylcynaropicrin (4), integrifolin-3-O- } \beta \text {-D-glucopyranoside (10), } \\
\text { ixerisoside A (14), 15, ixerin N (21), ixerin M (22), } \\
\text { 8 } \beta \text {-hydroxy-4 } \beta, 15 \text {-dihydrozaluzanin C (26), 40, 8-epi-isolipidiol (42), } \\
\text { 8-epi-isolipidiol-3-O- } \beta \text { - } D \text {-glucopyranoside (50) }\end{array}$ & {$[66]$} \\
\hline C. biennis & Poland & roots & ixerin F (41) & [67] \\
\hline C. cameroonica & Cameroon & aerial parts & $\begin{array}{c}3 \beta, 9 \beta \text {-dihydroxyguaian- } 4(15), 10(14), 11(13) \text {-trien-6,12-olide (2), } \\
\text { 8-desacylcynaropicrin (3), } \\
8 \alpha \text {-hydroxy-4 } \alpha(15), 11 \beta(13) \text {-tetrahydrozaluzanin C (47) }\end{array}$ & [30] \\
\hline \multirow[t]{2}{*}{ C. capillaris } & Poland & roots & $\begin{array}{c}\text { integrifolin (8-epi-desacylcynaropicrin) (4), } \\
\text { integrifolin-3-O- } \beta \text { - } D \text {-glucopyranoside }(\mathbf{1 0}), \mathbf{1 6}, \mathbf{1 7}, 8 \text {-epi-isolipidiol (42), } \\
\text { 8-epi-isolipidiol-3-O- } \beta \text { - } D \text {-glucopyranoside (50) }\end{array}$ & [68] \\
\hline & Poland & aerial parts & $\begin{array}{c}\text { integrifolin (8-epi-desacylcynaropicrin) (4), 8-epi-isolipidiol (42), } \\
\text { 8-epi-isoamberboin (49) }\end{array}$ & {$[69,70]$} \\
\hline C. commutata & Greece & aerial parts & $\begin{array}{l}\text { integrifolin-3-O- } \beta \text { - } D \text {-glucopyranoside (10), 8-epi-grosheimin (24), } \\
\text { 8-epi-isolipidiol (42), 3-acetyl-8-epi-isolipidiol (43), 8-epi-isoamberboin (49) }\end{array}$ & [71] \\
\hline C. conyzifolia & Poland & roots & $\begin{array}{c}\text { 8 } \beta \text {-hydroxy-4 } \beta, 15 \text {-dihydrozaluzanin C (26), } 4 \\
\beta, 15,11 \beta, 13 \text {-tetrahydrozaluzanin-C-3-O- } \beta \text {-glucopyranoside (31), } \\
\text { 8-epi-isolipidiol (42), 8-epi-isolipidiol-3O- } \beta \text { - } D \text {-glucopyranoside (50) }\end{array}$ & [72] \\
\hline C. crocea & Mongolia & aerial parts & $\begin{array}{l}\text { integrifolin (8-epi-desacylcynaropicrin) (4), } 11 \beta, 13 \text {-dihydrointegrifolin (36), } \\
\text { 8-epi-isoamberboin (49) }\end{array}$ & [73] \\
\hline C. dioscoridis & Greece & aerial parts & $\begin{array}{c}\text { integrifolin (4), integrifolin-3O- } \beta \text { - } D \text {-glucopyranoside (10), } \\
\text { 8-epi-grosheimin (24), crepiside C (29), } 11 \beta, 13 \text {-dihydrointegrifolin (36), } \\
11 \beta, 13 \text {-dihydrointegrifolin-3-O- } \beta \text { - } D \text {-glucopyranoside (38), } \\
11 \beta, 13 \text {-dihydro-8- } \beta \text {-hydroxy- } 4 \alpha, 15 \text {-dihydrozaluzanin } \\
\text { 8-epi-isolipidiol-3-O- } \beta \text { - } D \text {-glucopyranoside (50), } \\
\text { 8-3-O- } \beta \text { - } D \text {-glucopyranoside }(51), 11 \beta, 13 \text {-dihydrocrepiside C (52), } \\
\text { 8-deoxylactucin (56) }\end{array}$ & {$[74]$} \\
\hline C. foetida & Poland & roots & $\begin{array}{c}9 \alpha \text {-hydroxy-11 } \beta, 13 \text {-dihydrozaluzanin (35), 11 } \beta, 13 \text {-dihydroglucozaluzanin } \\
\text { C (37), ixerin F (41), 44, } 45\end{array}$ & [75] \\
\hline
\end{tabular}


Table 2. Cont.

\begin{tabular}{|c|c|c|c|c|}
\hline Taxa & Origin & Parts & Sesquiterenes & Ref. \\
\hline C. incana & Greece & aerial parts & $\begin{array}{l}\text { crepiside } \mathrm{E}(9) \text {, grosheimin }(25) \text {, crepiside } \mathrm{D}(28) \text {, taraxinic } \\
\text { acid- } 1^{\prime} O-\beta \text { - } D \text {-glucopyranoside }(72) \text {, taraxinic acid }(73)\end{array}$ & [76] \\
\hline C. japonica & Japan & whole plant & $\begin{array}{c}\text { glucozaluzanin C (8), crepiside E (9), crepiside G (11), crepiside B (13), } \\
\text { crepiside A (18), } \text {, crepiside H (19), crepiside I (20), crepiside D (28), } \\
\text { crepiside C (29), crepiside F (30) }\end{array}$ & [77] \\
\hline C. lacera & Italy & aerial parts & $\begin{array}{c}\text { integrifolin-3-O- } \beta \text { - } D \text {-glucopyranoside (10), 8-epi-grosheimin (24), } \\
\text { 8- } \beta \text {-hydroxydehydrozaluzanin C (5), } \\
\text { 11 } \beta, 13 \text {-dihydrointegrifolin-3- } O-\beta \text { - } D \text {-glucopyranoside (38) }\end{array}$ & [47] \\
\hline $\begin{array}{l}\text { C. leontodon- } \\
\text { toides }\end{array}$ & Italy & aerial parts & $\begin{array}{l}\text { 8-deoxylactucin (56), lactucin (57), 11 } \beta, 13 \text {-dihydro-8-deoxylactucin (58), } \\
\text { 15-deoxylactucin-8-sulfate (60), 11 } \beta, 13 \text {-dihydrolactucin-8-sulfate (61) }\end{array}$ & [78] \\
\hline C. micrantha & Egypt & aerial parts & $\begin{array}{c}\text { 8-desacylcynaropicrin (3), 11 } \beta, 13 \text {-dihydrointegrifolin (36), } \\
\text { 8-epi-isolipidiol (42) }\end{array}$ & [79] \\
\hline
\end{tabular}

integrifolin-3-O- $\beta$-D-glucopyranoside (10), 16, 17, 8-epi-grosheimin (24), $11 \beta, 13$-dihydrozaluzanin C (34),

C. mollis Poland roots 11 $\beta, 13$-dihydrointegrifolin-3- $O-\beta$ - $D$-glucopyranoside (38), 40, ixerin F (41), 8-epi-isolipidiol (42),

$9 \alpha$-hydroxy-4b,15,11 $\beta, 13$-tetra-hydro-dehydrozaluzanin C (48),

8-epi-isolipidiol-3-O- $\beta$-D-glucopyranoside (50), picriside B (71)

\begin{tabular}{ccccc}
\hline C. multicaulis & Kazakh. & aerial parts & crepidioside A (59) & [81] \\
\hline \multirow{3}{*}{ C. napifera } & China & roots & napiferoside (65) & [82] \\
\cline { 2 - 5 } & China & roots & $\begin{array}{c}\text { taraxinic acid-1'O- } \beta \text { - } D \text {-glucopyranoside (72), 11 } \beta \text {,13-dihydro-taraxinic } \\
\text { acid-1'O- } \beta \text {-D-glucopyranoside (74) }\end{array}$ & [83] \\
\hline
\end{tabular}

$9 \alpha$-hydroxy-3-deoxyzaluzanin C (1), macrocliniside A (7),

integrifolin-3O- $\beta$ - $D$-glucopyranoside (10), pannonicoside (23),

C. pannonica Poland roots $8 \beta$-hydroxy- $4 \beta, 15$-dihydrozaluzanin $C-3 O-\beta$ - $D$-glucopyranoside (32),

8 - $\beta$-hydroxy-4 $\alpha, 15$-dihydrozaluzanin C-3-O- $\beta$ - $D$-glucopyranoside (33), [84]

40, ixerin F (41), 8-epi-isolipidiol-3O- $\beta$-D-glucopyranoside (50),

$11 \beta, 13$-dihydro- $8-\beta$-hydroxy- $4 \alpha, 15$-dihydrozaluzanin

C-3-O- $\beta$ - $D$-glucopyranoside (51)

\begin{tabular}{|c|c|c|c|c|}
\hline C. pulchra & Poland & roots & $\begin{array}{c}\text { diaspanosise A (6), macrocliniside A (7), glucozaluzanin C (8), } \\
\text { 8-epi-isoamberboin (49) }\end{array}$ & [85] \\
\hline \multirow{2}{*}{ C. pygmaea } & Italy & whole plant & $\begin{array}{l}\text { 1,2-4,5-tetrahydro-11-nor-11-hydroxy- } \Delta^{7,11} \text {-santonin (63), } \\
\text { 4,5-dihydro-11-nor-11-hydroxy- } \Delta^{7,11} \text {-santonin (64) }\end{array}$ & {$[86,87]$} \\
\hline & Poland & roots & $\begin{array}{l}\text { macrocliniside A (7), integrifolin-3O- } \beta \text { - } D \text {-glucopyranoside }(\mathbf{1 0}), \mathbf{1 6}, \mathbf{1 7}, \\
8 \beta \text {-hydroxy- } 4 \beta, 15 \text {-dihydrozaluzanin } C-3 O-\beta-D \text {-glucopyranoside (32), } \\
\text { ixerin F (41), 8-epi-isolipidiol-3O- } \beta \text { - } D \text {-glucopyranoside (50) }\end{array}$ & [88] \\
\hline C. rhoeadifolia & Poland & roots & $11 \beta, 13$-dihydroglucozaluzanin C (37), ixerin F (41), 44, 45 & [89] \\
\hline \multirow{3}{*}{ C. sancta } & Jordan & aerial parts & $\begin{array}{l}\text { 3-oxo-di-nor-eudesm-4-en-6 } \alpha \text {-hydroxy-11-oic acid (66), } \\
\text { 3-oxo-6 } \beta \text {-hydroxy- } \gamma \text {-costic acid (67), 3-oxo- } \gamma \text {-costic acid (68), } \\
\text { 3-oxo- } \gamma \text {-costic acid methyl ester }(69),(6 R, 9 S) \text {-roseoside }(70)\end{array}$ & [90] \\
\hline & Jordan & aerial parts & 3-oxo- $\gamma$-costic acid (68), 3-oxo- $\gamma$-costic acid methyl ester (69) & [91] \\
\hline & Italy & aerial parts & $\begin{array}{c}\text { 8-deoxylactucin (56), } 11 \beta, 13 \text {-dihydro-8-deoxylactucin (58), } \\
\text { lactucopicrin (62) }\end{array}$ & [78] \\
\hline C. setosa & Poland & roots & $\begin{array}{l}9 \alpha \text {-hydroxy-11 } \beta, 13 \text {-dihydrozaluzanin (35), } 11 \beta, 13 \text {-dihydroglucozaluzanin } \\
\mathrm{C}(37), 8 \alpha \text {-hydroxy-11 } \beta, 13 \text {-dihydroglucozaluzanin (39), ixerin F (41) }\end{array}$ & [92] \\
\hline C. sibirica & Poland & roots & $\begin{array}{c}\text { glucozaluzanin } C(8) \text {, integrifolin-3O- } \beta \text { - } D \text {-glucopyranoside (10), } \\
\text { 8-epi-isoamberboin }(49)\end{array}$ & [93] \\
\hline \multirow[t]{2}{*}{ C. tectorum } & Poland & roots & $\begin{array}{l}\text { glucozaluzanin } C(8) \text {, tectoroside }(\mathbf{1 2}), \mathbf{1 6}, \mathbf{1 7} \text {, ixerin F (41), isolipidiol (46), } \\
\text { 8-epi-isolipidiol-3-O- } \beta \text { - } D \text {-glucopyranoside (50) }\end{array}$ & {$[94,95]$} \\
\hline & Kazakh. & aerial parts & integrifolin (8-epi-desacylcynaropicrin) (4), 8-epi-isolipidiol (42) & {$[81,96]$} \\
\hline
\end{tabular}


Table 2. Cont.

\begin{tabular}{|c|c|c|c|c|}
\hline Taxa & Origin & Parts & Sesquiterenes & Ref. \\
\hline C. tingitana & Spain & $\begin{array}{l}\text { sub aerial } \\
\text { parts }\end{array}$ & integrifolin-3O- $\beta$ - $D$-glucopyranoside (10), ixerisoside A (14) & [97] \\
\hline C. vesicaria & Italy & aerial parts & 8-deoxylactucin (56), 11 $\beta, 13$-dihydro-8-deoxylactucin (58) & [78] \\
\hline C. virens & Italy & flowers & 8-epi-grosheimin (24) & [98] \\
\hline C. zacintha & Poland & roots & 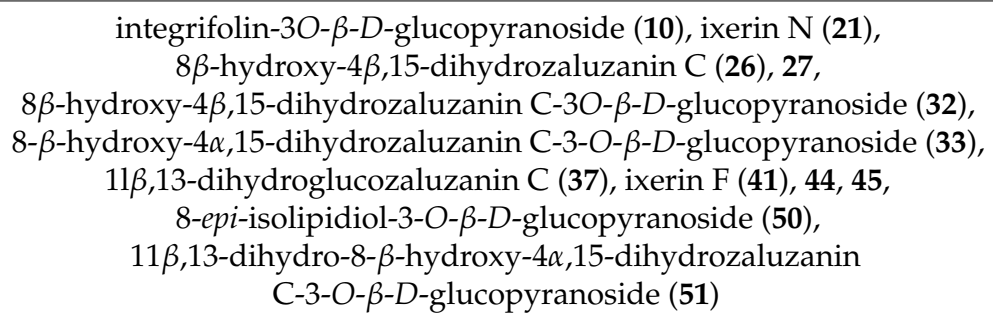 & [99] \\
\hline
\end{tabular}

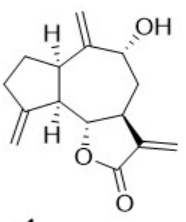

1

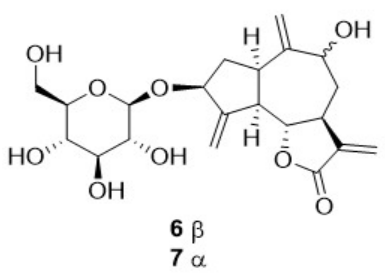<smiles>C=C1C(=O)O[C@H]2C1CC(O)C(=C)[C@H]1CC(O)C(=C)[C@@H]12</smiles><smiles>C=C1C[C@@H](O)[C@H]2C(=C)C(=O)O[C@H]2[C@H]2C(=C)[C@@H](O)C[C@@H]12</smiles>

$3 \alpha$<smiles>C=C1C(=O)O[C@@H]2[C@@H]1[C@@H](O)CC(=C)[C@@H]1CC(=O)C(=C)[C@@H]21</smiles>

5<smiles>[R]C1CC(=C)[C@@H]2C[C@@H](O[C@@H]3O[C@H](CO)[C@@H](O)[C@H](O)[C@H]3O)C(=C)[C@H]2[C@H]2OC(=O)C(=C)[C@H]12</smiles>

$\begin{array}{ll}9 & \mathrm{R}=\alpha \mathrm{OH} \\ 10 & \mathrm{R}=\beta \mathrm{OH}\end{array}$

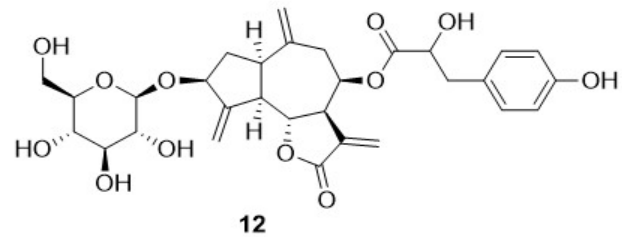<smiles>C=C1C(=O)O[C@H]2C1CCC(=C)[C@H]1C(=C)C(O[C@@H]3OC(CO)[C@H](OC(=O)Cc4ccc(O)cc4)[C@H](O)[C@H]3O)C[C@H]12</smiles><smiles>[R2]Oc1ccc(CC(=O)O[C@H]2CC(=C)[C@@H]3C[C@H](O[C@@H]4O[C@H](CO)[C@@H](O)[C@H](O)[C@H]4O)C(=C)[C@H]3[C@H]3OC(=O)C(=C)[C@@H]23)cc1</smiles>

$14 \mathrm{R}=\mathrm{H}$

$15 \mathrm{R}=\mathrm{CH}_{3}$<smiles>CC(=O)Cc1ccc(O)cc1</smiles><smiles>[R]C1CC(=C)[C@H]2C[C@@H](O[C@@H]3O[C@H](COC(C)=O)[C@@H](O)[C@H](O)[C@H]3O)C(=C)[C@H]2[C@@H]2OC(=O)C(=C)[C@H]12</smiles>

$18 \mathrm{R}=\mathrm{H}$

$19 \mathrm{R}=\alpha \mathrm{OH}$

$20 \mathrm{R}=\beta \mathrm{OH}$<smiles>[R]C(O)(C(C)=O)C(=O)O[C@H]1CC(=C)[C@H]2C[C@H](O[C@@H]3O[C@H](CO)[C@@H](O)[C@H](O)[C@H]3O)C(=C)[C@H]2[C@H]2OC(=O)C(=C)[C@@H]12</smiles>

$16 \mathrm{R}=\mathrm{CH}_{3}$

$17 \mathrm{R}=\mathrm{CH}_{2} \mathrm{CH}_{3}$

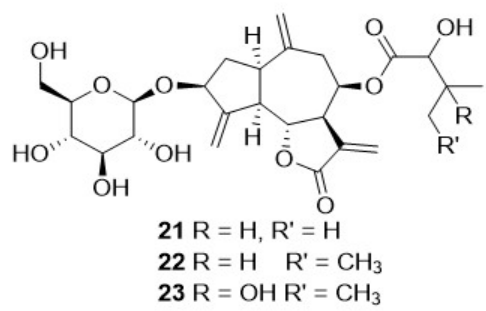

Figure 3. 4,15,10,14,11,13-dehydro guaianolides from Crepis taxa. 
<smiles>C=C1C(=O)O[C@@H]2[C@@H]1[C@H](O)CC(=C)[C@H]1CC(=O)[C@@H](C)[C@@H]12</smiles>

$24 \beta$

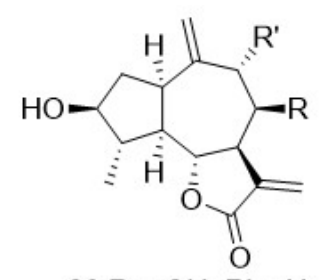

$26 \mathrm{R}=\mathrm{OH}, \mathrm{R}^{\prime}=\mathrm{H}$

$27 \mathrm{R}=\mathrm{H}, \mathrm{R}^{\prime}=\mathrm{OH}$<smiles>C=C1C(=O)O[C@@H]2[C@@H]1[C@@H](OC(=O)Cc1ccc(O)cc1)CC(=C)[C@H]1CC(O[C@@H]3O[C@H](CO)[C@@H](O)[C@H](O)[C@H]3O)=C(C)[C@@H]12</smiles>

30<smiles>[R]C1CC(=C)[C@H]2CC(O[C@@H]3O[C@H](CO)[C@@H](O)[C@H](O)[C@H]3O)=C(C)[C@@H]2[C@H]2OC(=O)C(=C)[C@H]12</smiles>

$28 \mathrm{R}=\alpha \mathrm{OH}$

$29 \mathrm{R}=\beta \mathrm{OH}$<smiles>[R]C1CC(=C)[C@H]2C[C@@H](O[C@@H]3O[C@H](CO)[C@@H](O)[C@H](O)[C@H]3O)[C@H]([R])[C@H]2[C@H]2OC(=O)C(=C)[C@H]12</smiles>

$31 \mathrm{R}=\alpha \mathrm{CH}_{3}, \mathrm{R}^{\prime}=\mathrm{H}$

$32 \mathrm{R}=\alpha \mathrm{CH}_{3}, \mathrm{R}^{\prime}=\mathrm{OH}$

$33 \mathrm{R}=\mathrm{\beta CH}_{3}, \mathrm{R}^{\prime}=\mathrm{OH}$

Figure 4. 10,14,11,13-dehydro guaianolides from Crepis taxa.<smiles>[R]C1C(=C)[C@H]2C[C@H](O)C(=C)[C@H]2[C@@H]2OC(=O)[C@H](C)C12</smiles>

$34 \mathrm{R}=\mathrm{H}, \mathrm{R}^{\prime}=\mathrm{H}$

$35 \mathrm{R}=\mathrm{H}, \mathrm{R}^{\prime}=\mathrm{OH}$

$36 \mathrm{R}=\mathrm{OH}, \mathrm{R}^{\prime}=\mathrm{H}$<smiles>[R]C1CC(=C)[C@H]2C[C@@H](O[C@@H]3O[C@H](CO)[C@@H](O)[C@H](O)[C@H]3O)C(=C)[C@@H]2[C@@H]2OC(=O)[C@H](C)[C@H]12</smiles>

$37 \mathrm{R}=\mathrm{H}$

$38 \mathrm{R}=\beta \mathrm{OH}$

$39 \mathrm{R}=\alpha \mathrm{OH}$

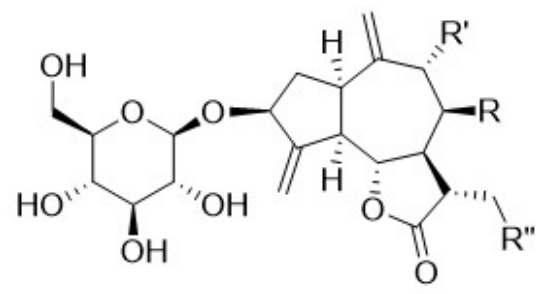

$40 \mathrm{R}=\mathrm{OH}, \mathrm{R}^{\prime}=\mathrm{H}, \mathrm{R}^{\prime \prime}=\mathrm{OCH}_{3}$ $41 \mathrm{R}=\mathrm{H}, \mathrm{R}^{\prime}=\mathrm{OH}, \mathrm{R}^{\prime \prime}=\mathrm{H}$

Figure 5. 4,15,10,14-dehydro guaianolides from Crepis taxa.<smiles>[R20]C1C[C@H]2C(=C)C[C@@H](O)[C@@H]3[C@@H](C)C(=O)O[C@@H]3[C@@H]2[C@H]1C</smiles>

$42 \mathrm{R}=\mathrm{H}$

$43 \mathrm{R}=\mathrm{Ac}$<smiles>C=C1CC(O)[C@H]2[C@@H](C)C(=O)O[C@@H]2[C@H]2[C@@H](C)C(=O)C[C@@H]12</smiles>

49

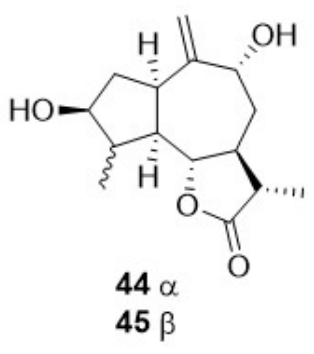<smiles>C=C1CC(O)[C@@H]2[C@@H](OC(=O)[C@@H]2C)[C@@H]2[C@H]1CC(O)[C@@H]2C</smiles>

$47 \beta$<smiles>C=C1C[C@H](O)[C@@H]2[C@@H](OC(=O)[C@@H]2C)[C@@H]2[C@@H]1CC(O[C@@H]1O[C@H](CO)[C@@H](O)[C@H](O)[C@H]1O)[C@@H]2C</smiles>

$50 \alpha$<smiles>C=C1C2CC(=O)[C@@H](C)[C@H]2C2OC(=O)[C@@H](C)[C@@H]2C[C@H]1O</smiles>

48
52

Figure 6. 10,14-dehydro guaianolides from Crepis taxa. 


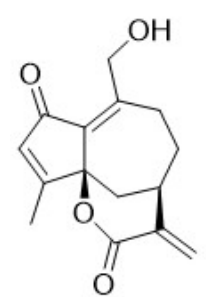

53

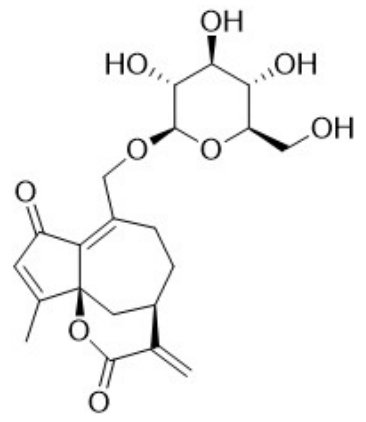

54

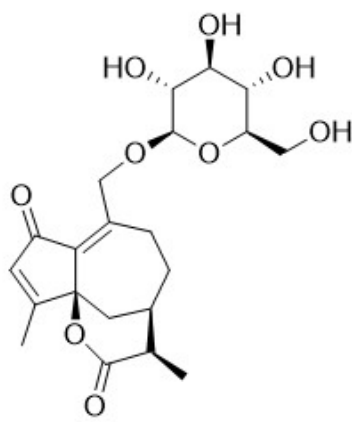

55

Figure 7. Hypocretenolides from Crepis taxa.<smiles>[R]C1CC(C)=C2C(=O)C=C(CO)[C@@H]2[C@@H]2OC(=O)C(=C)[C@H]12</smiles>

$56 \mathrm{R}=\mathrm{H}$ $57 \mathrm{R}=\mathrm{OH}$<smiles>C=C1C(=O)O[C@H]2[C@H]1[C@@H](OS(=O)(=O)O)CC(C)=C1C(=O)C=C(C)[C@H]12</smiles>

60<smiles>CC1=C2C(=O)C=C(CO)[C@H]2[C@H]2OC(=O)[C@H](C)[C@H]2CC1</smiles>

58

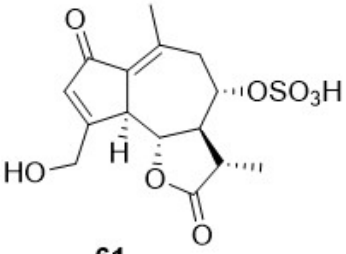

61
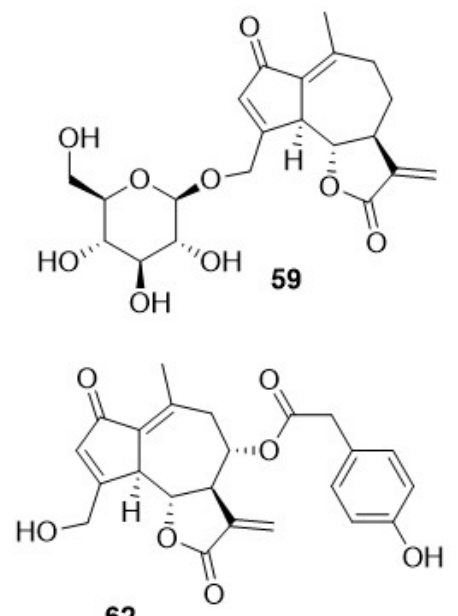

Figure 8. Lactucin type guaianolides from Crepis taxa.<smiles>C[C@H]1C(=O)CCC2(C)CCC3=C(O)C(=O)O[C@@H]3[C@H]12</smiles>

63<smiles>C[C@H]1C(=O)C=CC2(C)CCC3=C(O)C(=O)OC3[C@H]12</smiles>

64<smiles>CC1=C2CC(C(C)C(=O)O)C(C(=O)OC(C)C)C=C2CCC1</smiles>

65<smiles>CC1=C2C(O)C(C(=O)O)CCC2(C)CCC1=O</smiles>

66<smiles>C=C(C(=O)O)[C@H]1CC[C@@]2(C)CCC(=O)C(C)=C2[C@H]1O</smiles>

67<smiles>[R]OC(=O)C(=C)C1CCC2(C)CCC(=O)C(C)=C2C1</smiles>

$68 \mathrm{R}=\mathrm{H}$

$69 \mathrm{R}=\mathrm{CH}_{3}$
70<smiles>C=C1C(=O)O[C@@H](/C=C2CC/C=C\CC/C=C/2CO)[C@@H]1C</smiles><smiles>[R2]C1OC(=O)C(=C)[C@@H]1/C=C(\C)CC/C=C\CC</smiles><smiles>[R]C1=CCCC(C)=CC[C@H]1OC(=O)C(C)C</smiles>

$74 \mathrm{R}=\mathrm{COOG} \mathrm{lc}$

Figure 9. Other sesquiterpenoids from Crepis taxa.

It is noteworthy that all guaianolides belonging to the Costus lactone type, grouped on the basis of their exocyclic double bonds (Figures 3-6), have a C-6/C-12 $\gamma$-lactone with a trans junction and that, apart from $9 \alpha$-hydroxy-3-deoxyzaluzanin C (1) [73], they all possess an oxygenated function at $C-3$. Other oxygenated functions can occur on C-8 
and/or C-9, but never on C-1, C-2, C-5, and C-11. The three hypocretenolides (53-55) (Figure 7) were detected only in the roots of C. aurea (L.) Cass [65], whereas the presence of lactucin derivatives (Figure 8 ) has been observed only in aerial parts of C. dioscoridis L. (8-deoxylactucin, 56) [74], C. leontodontoides All. [78], and C. sancta (L.) Bornm. [78].

A very small numer of sesquiterpenes with different skeletons-eudesmane (63-69), and germacrane (71-74) (Table 2, Figure 9)-have been detected in Crepis species. The former occurred in C. pygmaea L. [86,87], C. napifera (Franch.) Babcock [82], and C. sancta (L.) Bornm. [90,91], whereas germacranolide was present only in C. mollis (Jacq.) Asch. [80], C. incana Sm. [76], and C. napifera (Franch.) Babcock [83].

\subsection{Flavonoids}

The occurrence of flavonoids, detected in the twenty-six Crepis taxa studied so far, is reported in Table 3, and their structures are depicted in Figure 10.

Table 3. Flavonoids from Crepis taxa.

\begin{tabular}{|c|c|c|c|c|}
\hline Taxa & Origin & Parts & Sesquiterenes & Ref. \\
\hline C. alpestris & Austria & aerial parts & $\begin{array}{c}\text { luteolin (76), luteolin 7-O-glucoside (80), luteolin } \\
\text { 7-O-glucuronide (81), luteolin 7-O-gentiobioside (82), } \\
\text { luteolin } 4^{\prime} \text {-O-glucoside (83) }\end{array}$ & [100] \\
\hline C. aurea & Austria & aerial parts & $\begin{array}{l}\text { luteolin (76), luteolin 7-O-glucoside (80), luteolin } \\
\text { 7-O-glucuronide (81) }\end{array}$ & [100] \\
\hline C. biennis & Austria & aerial parts & $\begin{array}{l}\text { luteolin (76), luteolin 7-O-glucoside (80), luteolin } \\
\text { 7-O-glucuronide (81), luteolin 7-O-gentiobioside (82) }\end{array}$ & [100] \\
\hline \multirow{3}{*}{ C. capillaris } & Austria & aerial parts & luteolin (76), luteolin 7-O-glucoside (80), & [100] \\
\hline & Spain & aerial parts & $\begin{array}{l}\text { luteolin (76), luteolin 7-O-glucoside (80), luteolin } \\
4^{\prime} \text {-O-glucoside (83) }\end{array}$ & [101] \\
\hline & New Zeland & flowers & $\begin{array}{l}\text { luteolin (76), luteolin 7-O-glucoside (80), luteolin } \\
\text { 7-O-glucuronide (81) }\end{array}$ & [102] \\
\hline C. commutata & Greece & aerial parts & luteolin (76), luteolin 7-O-glucuronide (81) & [71] \\
\hline C. conyzifolia & Austria & aerial parts & $\begin{array}{l}\text { luteolin (76), luteolin 7-O-glucoside (80), luteolin } \\
\text { 7-O-glucuronide (81), luteolin } 4^{\prime}-O \text {-glucoside (79) }\end{array}$ & [100] \\
\hline C. dioscoridis & Greece & aerial parts & apigenin- $4^{\prime}$-O-glucoside (83), isorhamnetin (85) & [74] \\
\hline C. divaricata & & & luteolin (76), quercetin (84) & [103] \\
\hline C. foetida & Italy & aerial parts & $\begin{array}{c}\text { luteolin (76), luteolin 7-O-glucoside (80), luteolin } \\
\text { 7-O-gentiobioside (82) }\end{array}$ & [100] \\
\hline \multirow{2}{*}{$\begin{array}{l}\text { C. foetida ssp. } \\
\text { rhoeadifolia }\end{array}$} & Turkey & flowers & apigenin (75), quercetin $(84),(+)$-catechin $(94)$ & [104] \\
\hline & Turkey & aerial parts & luteolin (76) & [105] \\
\hline C. froelichiana. & Italy & aerial parts & $\begin{array}{l}\text { luteolin (76), luteolin 7-O-glucoside (80), luteolin } \\
\text { 7-O-glucuronide (81), luteolin } 4^{\prime} \text {-O-glucoside (83) }\end{array}$ & [100] \\
\hline C. incana & Greece & aerial parts & $\begin{array}{l}\text { luteolin (76), luteolin 7-O-glucoside (80), quercetin } \\
\text { 3-glucoside (93) }\end{array}$ & [76] \\
\hline $\begin{array}{l}\text { C. jacquinii ssp. } \\
\text { kerneri }\end{array}$ & Italy & aerial parts & luteolin (76), luteolin 7-O-glucoside (80) & [100] \\
\hline C. japonica & & & luteolin-7-O-glucoside (80) & {$[45,106]$} \\
\hline C. micrantha & Egypt & aerial parts & apigenin (75), luteolin (76) & [79] \\
\hline C. mollis & Austria & aerial parts & $\begin{array}{l}\text { luteolin (76), luteolin 7-O-glucoside (80), luteolin } \\
\text { 7-O-glucuronide (81), luteolin 7-O-gentiobioside (82) }\end{array}$ & [100] \\
\hline C. nemausensis & Italy & aerial parts & $\begin{array}{l}\text { luteolin (76), luteolin 7-O-glucoside (80), luteolin } \\
\text { 7-O-glucuronide (81), luteolin } 4^{\prime}-O \text {-glucoside (83) }\end{array}$ & [100] \\
\hline
\end{tabular}


Table 3. Cont.

\begin{tabular}{|c|c|c|c|c|}
\hline Taxa & Origin & Parts & Sesquiterenes & Ref. \\
\hline C. paludosa & Germany & aerial parts & $\begin{array}{l}\text { luteolin (76), luteolin 7-O-glucoside (80), luteolin } \\
\text { 7-O-glucuronide (81), luteolin } 4^{\prime} \text {-O-glucoside (83) }\end{array}$ & [100] \\
\hline \multirow[t]{2}{*}{ C.pygmaea } & Italy & aerial parts & $\begin{array}{l}\text { luteolin (76), luteolin 7-O-glucoside (80), luteolin } \\
\text { 7-O-gentiobioside (82), luteolin 7-O-glucuronide (81) }\end{array}$ & [100] \\
\hline & & & luteolin (76), quercetin (84) & [103] \\
\hline C. rhaetica & Austria & aerial parts & $\begin{array}{c}\text { luteolin (76), luteolin 7-O-glucoside (80), luteolin } \\
\text { 7-O-glucuronide (81) }\end{array}$ & [100] \\
\hline \multirow[b]{2}{*}{ C. sancta } & Jordan & aerial parts & kumatakenin (86), penduletin (87) & {$[90]$} \\
\hline & Jordan & aerial parts & $\begin{array}{l}\text { kumatakenin (86), penduletin (87), pachypodol (88), } \\
\text { jaceidin (89), chrysosplentin (90), casticin (91) } \\
\text { 3,5,7-tri-O-methyl-6-methoxy-kaempferol (92) }\end{array}$ & [91] \\
\hline C. senecioides & & & luteolin (76), isoetin (77), scutellarein (78) & [107] \\
\hline C. tectorum & & & isoetin (77), scutellarein (78) & [107] \\
\hline C. terglouensis & Switzerland & aerial parts & $\begin{array}{l}\text { luteolin (76), luteolin 7-O-glucoside (80), luteolin } \\
\text { 7-O-glucuronide (81), luteolin 7-O-gentiobioside (82) }\end{array}$ & [100] \\
\hline C. tingitana & Spain & aerial parts & $\begin{array}{l}\text { luteolin (76), luteolin 7-O-glucoside (80), luteolin } \\
\text { 7-O-glucuronide (81), luteolin } 4^{\prime} \text {-O-glucoside (83) }\end{array}$ & [100] \\
\hline C. vesicaria & Spain & aerial parts & $\begin{array}{l}\text { luteolin (76), luteolin 7-O-glucoside (80), luteolin } \\
\text { 4'-O-glucoside (83) }\end{array}$ & {$[101,108]$} \\
\hline
\end{tabular}

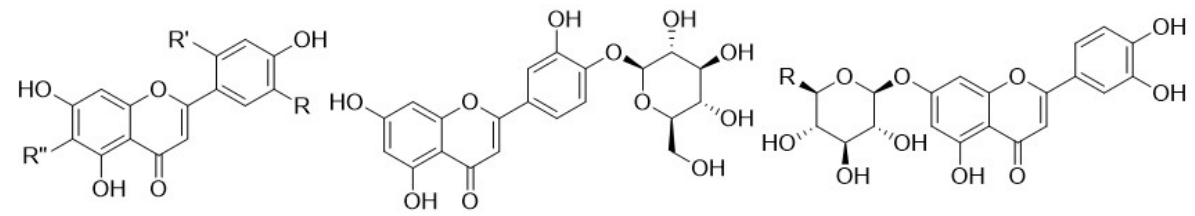

$75 \mathrm{R}=\mathrm{H}, \mathrm{R}^{\prime}=\mathrm{R}^{\prime \prime}=\mathrm{H}$

$76 \mathrm{R}=\mathrm{OH}, \mathrm{R}^{\prime}=\mathrm{R}^{\prime \prime}=\mathrm{H}$

79

$80 \mathrm{R}=\mathrm{CH}_{2} \mathrm{OH}$

$77 \mathrm{R}=\mathrm{R}^{\prime}=\mathrm{OH}, \mathrm{R}^{\prime \prime}=\mathrm{H}$

$78 \mathrm{R}=\mathrm{R}^{\prime}=\mathrm{H}, \mathrm{R}^{\prime \prime}=\mathrm{OH}$
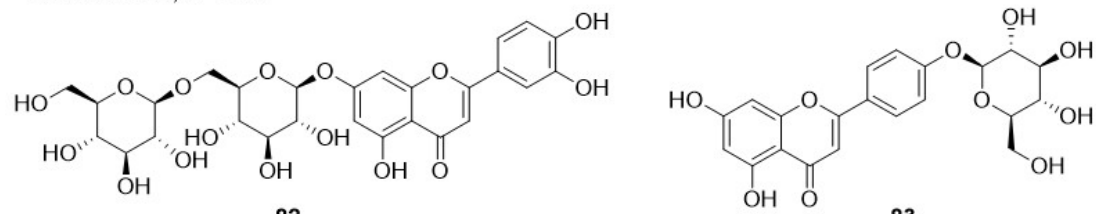

82

83<smiles>O=c1c(O)c(-c2ccc(O)c(O)c2)oc2cc(O)cc(O)c12</smiles>

$84 \mathrm{R}=\mathrm{H}$

$85 \mathrm{R}=\mathrm{CH}_{3}$<smiles>[R]c1cc(-c2oc3cc([R20])c([R])c(O)c3c(=O)c2OC)ccc1O</smiles>

$86 \mathrm{R}=\mathrm{H}, \mathrm{R}^{\prime}=\mathrm{CH}_{3}, \mathrm{R}^{\prime \prime}=\mathrm{H}$ $87 \mathrm{R}=\mathrm{OCH}_{3}, \mathrm{R}^{\prime}=\mathrm{CH}_{3}, \mathrm{R}^{\prime \prime}=\mathrm{H}$ $88 \mathrm{R}=\mathrm{H}, \mathrm{R}^{\prime}=\mathrm{CH}_{3}, \mathrm{R}^{\prime \prime}=\mathrm{OCH}_{3}$ $89 \mathrm{R}=\mathrm{OCH}_{3}, \mathrm{R}^{\prime}=\mathrm{CH}_{3}, \mathrm{R}^{\prime \prime}=\mathrm{OCH}_{3}$ $90 \mathrm{R}=\mathrm{OCH}_{3}, \mathrm{R}^{\prime}=\mathrm{H}, \mathrm{R}^{\prime \prime} \mathrm{OCH}_{3}$<smiles>O=c1c(OC2O[C@H](CO)[C@@H](O)[C@H](O)C2O)c(-c2ccc(O)c(O)c2)oc2cc(O)cc(O)c12</smiles><smiles>[R20]c1ccc(-c2oc3cc(OC)c(O)c(O)c3c(=O)c2OC)cc1O</smiles>

$91 \mathrm{R}=\mathrm{H}, \mathrm{R}^{\prime}=\mathrm{CH}_{3}$ $92 \mathrm{R}=\mathrm{CH}_{3}, \mathrm{R}^{\prime}=\mathrm{H}$<smiles>Oc1cc(O)c2c(c1)O[C@H](c1ccc(O)c(O)c1)C(O)C2</smiles>

Figure 10. Flavonoids from Crepis taxa. 
In contrast to the large diversity of sesquiterpenes reported, only a limited number of flavonoids have been observed in the genus Crepis. These are mainly luteolin derivatives, with luteolin itself (76) being the most common one, present in twenty-one species. Additionally, luteolin-7-O-glucoside (80) and luteolin 7-O-glucuronide (81) are present in several species, eighteen and thirteen, respectively, whereas luteolin 7-O-gentiobioside (82) and luteolin $4^{\prime}$-O-glucoside (83) occur in few cases. Among the flavonols, the most common one is quercetin (84), present in C. divaricata Boss. \& Heldr, C. pygmaea L. [103] and C. foetida L. ssp. rhoeadifolia (Bieb.) Celak. [104].

Quite interesting is the research carried out by Zidorn et al. [102], who investigated the influence of the altitude of the collection site on the quantitative content of flavonoids within the flowering heads of C. capillaris (L.) Wallr. growing in New Zealand. The results showed a clear increase in flavonoid content in relation to the altitude. Similar data were observed for taxa of genus Leontodon collected in Europe [109]. It was hypothesised that higher altitude sites are exposed to higher peak UV-B levels, and therefore plants synthesize and store higher levels of flavonoids as UV-B protective compounds in flowering heads.

\subsection{Other Metabolites}

Derivatives of caffeic acid (98) are well-known antioxidant metabolites widely represented in several wild herbs of the Asteraceae family such as arnica (Arnica montana L.), chamomiles [Chamaemelum nobile (L.) All.], feverfew [Tanacetum parthenium (L.) Sch. Bip.], giant goldenrod (Solidago gigantea Aiton), milfoil (Myriophyllum ssp.), mugwort (Artemisia vulgaris L.), and tansy (Tanacetum vulgare L.) [110]. They are also present in many of the Crepis species studied so far, and their occurrence and structures are shown in Table 4 and Figure 9, respectively. The main metabolites of this class are caffeic acid (98), chlorogenic acid (104), caffeoyltartaric acid (105), 3,5-di-O-dicaffeoylquinic acid (106), 3,4-di-O-caffeoylquinic acid (107), cichoric acid (108), and rosmarinic acid (112), found, with different occurrence, in twenty Crepis taxa. Other metabolites detected in Crepis ssp., including free carboxylic acids (95-97, 100, 101, 126), lignans (121, 122), eugenyl derivatives $(\mathbf{1 1 4}, \mathbf{1 1 5})$, triterpenoids (127-130), steroids $(\mathbf{1 3 1}, \mathbf{1 3 2})$, etc., are reported in Table 4 and Figures 11 and 12.

Table 4. Other metabolites present in Crepis taxa.

\begin{tabular}{|c|c|c|c|c|}
\hline Taxa & Origin & Parts & Compounds & Ref. \\
\hline C. alpestris & Austria & aerial parts & $\begin{array}{l}\text { chlorogenic acid (104), caffeoyltartaric acid (105), } \\
\text { 3,5-di-O-dicaffeoylquinic acid (106), cichoric acid (108) }\end{array}$ & [100] \\
\hline C. aspera & Egypt & aerial parts & oleanolic acid (129), sitosterol 3-O- $\beta$ - $D$-glucopyranoside (131) & [64] \\
\hline \multirow{2}{*}{ C. aurea } & Poland & roots & $\begin{array}{c}\text { syringing (109), } \\
\text { 5-methoxy-eugenyl-4-O- } \beta \text {-glucopyranoside (114) }\end{array}$ & [66] \\
\hline & Austria & aerial parts & $\begin{array}{l}\text { chlorogenic acid (104), caffeoyltartaric acid (105), } \\
\text { 3,5-di-O-dicaffeoylquinic acid (106), cichoric acid (108) }\end{array}$ & [100] \\
\hline C. biennis & Austria & aerial parts & $\begin{array}{l}\text { chlorogenic acid (104), caffeoyltartaric acid (105), } \\
\text { 3,5-di-O-dicaffeoylquinic acid (106), cichoric acid (108) }\end{array}$ & [100] \\
\hline C. bocconi & Germany & sub aerial parts & $\begin{array}{l}\text { 4-hydroxybenzoic acid 4- } \beta \text {-D-glucopyranosyloxy-benzyl ester } \\
\text { (111) }\end{array}$ & [111] \\
\hline \multirow{3}{*}{ C. capillaris } & Austria & aerial parts & $\begin{array}{l}\text { chlorogenic acid (104), caffeoyltartaric acid (105), } \\
\text { 3,5-di-O-dicaffeoylquinic acid (106), cichoric acid (108) }\end{array}$ & [100] \\
\hline & Spain & aerial parts & $\begin{array}{c}\text { chlorogenic acid (104), 3,5-di-O-dicaffeoylquinic acid (106) } \\
\text { cichoriin (120) }\end{array}$ & [101] \\
\hline & New Zeland & flowers & $\begin{array}{l}\text { caffeic acid (98), chlorogenic acid (104), caffeoyltartaric acid } \\
(\mathbf{1 0 5}), 3,5-\text { di-O-caffeoylquinic acid (106), cichoric acid (108) }\end{array}$ & [102] \\
\hline
\end{tabular}


Table 4. Cont.

\begin{tabular}{|c|c|c|c|c|}
\hline Taxa & Origin & Parts & Compounds & Ref. \\
\hline C. commutata & Greece & aerial parts & $\begin{array}{l}p \text {-anisic acid (97), E-caffeic acid (98), } \\
p \text {-hydroxyphenylacetic acid (100) }\end{array}$ & [71] \\
\hline \multirow[t]{2}{*}{ C. conyzifolia } & Poland & roots & $\begin{array}{l}\text { 5-methoxy-eugenyl-4-O- } \beta \text {-glucopyranoside (114), } \\
\text { eugenyl-4-O- } \beta \text {-glucopyranoside (115), cichoriin (120) }\end{array}$ & {$[72]$} \\
\hline & Austria & aerial parts & chlorogenic acid (104), 3,5-di-O-dicaffeoylquinic acid (106) & [100] \\
\hline C. crocea & & & polysaccarhides & {$[35]$} \\
\hline C. dioscoridis & Greece & aerial parts & E-caffeic acid (98), ferulic acid (99), isosalicin (116) & {$[74]$} \\
\hline C. foetida & Italy & aerial parts & $\begin{array}{l}\text { chlorogenic acid (104), caffeoyltartaric acid (105), } \\
\text { 3,5-di-O-dicaffeoylquinic acid (106), cichoric acid (108) }\end{array}$ & [100] \\
\hline \multirow{3}{*}{$\begin{array}{l}\text { C. foetida ssp. } \\
\text { rhoeadifolia }\end{array}$} & Turkey & floers & $\begin{array}{l}\text { protocatechuic acid (95), gallic acid (96), caffeic acid (98), } \\
\text { chlorogenic acid (104), rosmarinic acid (112) }\end{array}$ & [104] \\
\hline & Turkey & aerial parts. & chlorogenic acid (104) & [105] \\
\hline & Turkey & roots & chlorogenic acid (104) & [105] \\
\hline C. froelichiana & Italy & aerial parts & $\begin{array}{l}\text { chlorogenic acid (104), caffeoyltartaric acid (105), } \\
\text { 3,5-di-O-dicaffeoylquinic acid (106), cichoric acid (108) }\end{array}$ & [100] \\
\hline \multirow{2}{*}{ C. incana } & Greece & aerial parts & $(3 S, 5 R)$-loliolide (123) & [76] \\
\hline & Greece & roots & oleanolic acid (129), lupeol (130) & [76] \\
\hline $\begin{array}{l}\text { C. jacquinii ssp. } \\
\text { kerneri }\end{array}$ & Italy & aerial parts & $\begin{array}{l}\text { chlorogenic acid (104), caffeoyltartaric acid (105), } \\
\text { 3,5-di-O-dicaffeoylquinic acid (106), cichoric acid (108) }\end{array}$ & [100] \\
\hline C. japonica & & & $\begin{array}{l}\text { 3,5-di-O-dicaffeoylquinic acid (106), 3,4-di-O-caffeoylquinic } \\
\text { acid (107) }\end{array}$ & {$[45,106]$} \\
\hline \multirow[b]{2}{*}{ C. lacera } & Italy & aerial parts & euphorbol acetate (127) & [112] \\
\hline & Italy & aerial parts & $\begin{array}{c}p \text {-hydroxy-benzyl 7-O- } \beta \text {-glucopyranoside (118), } \\
\text { pynoresynol (121) }\end{array}$ & {$[47]$} \\
\hline \multirow{2}{*}{ C. mollis } & Poland & roots & $\begin{array}{l}\text { 3-hydroxy-1-(4-hydroxy-3-methoxyphenyl)-1-propanone } \\
\text { (101), 5-methoxy-eugenyl-4-O- } \beta \text {-glucopyranoside (114) }\end{array}$ & {$[80]$} \\
\hline & Austria & aerial parts & $\begin{array}{l}\text { chlorogenic acid (104), caffeoyltartaric acid (105), } \\
\text { 3,5-di-O-dicaffeoylquinic acid (106), cichoric acid (108) }\end{array}$ & [100] \\
\hline C. napifera & China & roots & acetate taraxasterol (128) & [82] \\
\hline C. nemausensis & Italy & aerial parts & $\begin{array}{l}\text { chlorogenic acid (104), caffeoyltartaric acid (105), } \\
\text { 3,5-di-O-dicaffeoylquinic acid (106), cichoric acid (108) }\end{array}$ & [100] \\
\hline C. paludosa & Germany & aerial parts & $\begin{array}{l}\text { chlorogenic acid (104), caffeoyltartaric acid (105), } \\
\text { 3,5-di-O-dicaffeoylquinic acid (106), cichoric acid (108) }\end{array}$ & [100] \\
\hline C. pannonica & Poland & roots & $\begin{array}{c}\text { syringing (109), dihydrosyringin (110), } \\
\text { 5-methoxy-eugenyl-4-O- } \beta \text {-glucopyranoside (114), } \\
\text { eugenyl-4-O- } \beta \text {-glucopyranoside (115), } \\
\text { benzyl-O- } \beta \text {-glucopyranoside (117), } \\
\text { 3-indolecarbaldehyde (124), nicotinamide (125) }\end{array}$ & [84] \\
\hline C.pygmaea & Italy & aerial parts & $\begin{array}{l}\text { chlorogenic acid (104), caffeoyltartaric acid (105), } \\
\text { 3,5-di-O-dicaffeoylquinic acid (106), cichoric acid (108) }\end{array}$ & [100] \\
\hline C. rhaetica & Austria & aerial parts & $\begin{array}{l}\text { chlorogenic acid (104), 3,5-dicaffeoylquinic acid, } \\
\text { caffeoyltartaric acid (105), cichoric acid (108) }\end{array}$ & [100] \\
\hline C. rhoeadifolia & Poland & roots & $\begin{array}{l}\text { isosalicn (102), helicin(103), dehydrodiconyferyl alcohol } \\
\text { diacetate (119) }\end{array}$ & [41] \\
\hline C. terglouensis & Switzerland & aerial parts & $\begin{array}{l}\text { chlorogenic acid (104), caffeoyltartaric acid (105), } \\
\text { 3,5-di-O-dicaffeoylquinic acid (106), cichoric acid (108) }\end{array}$ & [100] \\
\hline C. tingitana & Spain & aerial parts & chlorogenic acid (104), 3,5-di-O-dicaffeoylquinic acid (106) & [100] \\
\hline
\end{tabular}


Table 4. Cont.

\begin{tabular}{ccccc}
\hline Taxa & Origin & Parts & Compounds & Ref. \\
\hline C. turczaniowii & & & phillyrin (122), acetate taraxasterol (128), $\beta$-sitosterol (II) (132) & {$[114]$} \\
\cline { 2 - 5 } & & & chlorogenic acid (104), 4,4'-dihydroxy-stilbene (113) & {$[101,108]$} \\
\hline C. vesicaria & Spain & aerial parts & caffeic acid (98), cichoric acid (108), quinic acid (126) & [61] \\
\hline $\begin{array}{c}\text { C. vesicaria ssp. } \\
\text { taraxicifolia }\end{array}$ & Portugal & aerial parts & (113] & \\
\hline
\end{tabular}

Some studies have been carried out on the composition of the fixed oils from seeds [115-118] and aerial parts [61,112] of Crepis ssp., and their composition is reported in Table 5. Several fixed oils are characterised by the presence of two unusual fatty acids, vernolic and crepenynic acid. Vernolic acid is used in the manufacture of plastic formulations and paints, whereas crepenynic acid is potentially a useful intermediate in the chemical synthesis and production of conjugated triene intermediates [115].<smiles>[R]c1cc(C(=O)O)cc([R])c1[R]</smiles>

$95 \mathrm{R}=\mathrm{R}^{\prime}=\mathrm{OH}, \mathrm{R}^{\prime \prime}=\mathrm{H}$ $96 \mathrm{R}=\mathrm{R}^{\prime}=\mathrm{R}^{\prime \prime}=\mathrm{OH}$ $97 \mathrm{R}=\mathrm{R}^{\prime \prime}=\mathrm{H}, \mathrm{R}^{\prime}=\mathrm{OCH}_{3}$<smiles>Oc1ccccc1COCl</smiles>

102

103<smiles>[R]Oc1cc(/C=C/C(=O)O)ccc1O</smiles>

$98 \mathrm{R}=\mathrm{H}$ $99 \mathrm{R}=\mathrm{CH}_{3}$<smiles>COc1cc(C(=O)CCO)ccc1O</smiles>

100
101<smiles>O=Cc1ccccc1OC(=O)Cl</smiles><smiles>O=C(/C=C/c1ccc(O)c(O)c1)O[C@H](C(=O)O)[C@@H](O)C(=O)/C=C/c1ccc(O)c(O)c1</smiles>

104<smiles>O=C(/C=C/c1ccc(O)c(O)c1)O[C@H]1C[C@](O)(C(=O)O)C[C@H](OC(=O)/C=C/c2ccc(O)c(O)c2)[C@H]1OC(=O)/C=C/c1ccc(O)c(O)c1</smiles><smiles>COc1cc(/C=C/CO)cc(OC)c1O[C@@H]1O[C@H](CO)[C@@H](O)[C@H](O)[C@H]1O</smiles>

108

109

110 dihydro<smiles>O=C(/C=C/C(=O)c1ccc(O)c(O)c1)OC(Cc1ccc(O)c(O)c1)C(=O)O</smiles><smiles>[R]c1cc(CC=C)cc(OC)c1O[C@@H]1O[C@H](CO)[C@@H](O)[C@H](O)[C@H]1O</smiles><smiles>[R]c1ccc(CO[C@@H]2O[C@H](CO)[C@@H](O)[C@H](O)[C@H]2O)c([R])c1</smiles>

$116 \mathrm{R}=\mathrm{OHR}^{\prime}=\mathrm{H}$

$117 \mathrm{R}=\mathrm{HR}^{\prime}=\mathrm{H}$

$118 \mathrm{R}=\mathrm{HR}^{\prime}=\mathrm{OH}$

Figure 11. Other metabolites from Crepis taxa. 


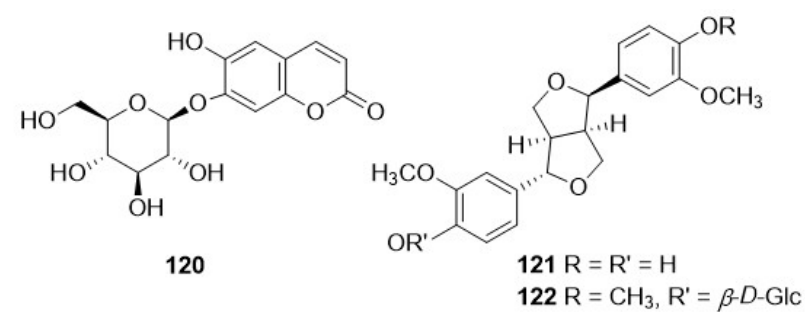<smiles>CC1(C)C[C@@H](O)C[C@]2(C)OC(=O)C=C12</smiles>

123<smiles>O=Cc1c[nH]c2ccccc12</smiles>

124<smiles>NC(=O)c1cccnc1</smiles>

125
$\mathrm{HO}$<smiles>O=C(O)C1(O)C[C@H](O)[C@H](O)[C@H](O)C1</smiles>

126

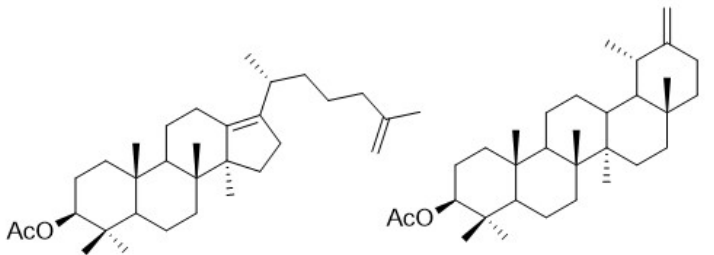

128<smiles>CC1(C)CCC2(C(=O)O)CC[C@H]3C(=CCC4[C@@]5(C)CC[C@@H](O)C(C)(C)C5CC[C@]43C)C2C1</smiles>

129

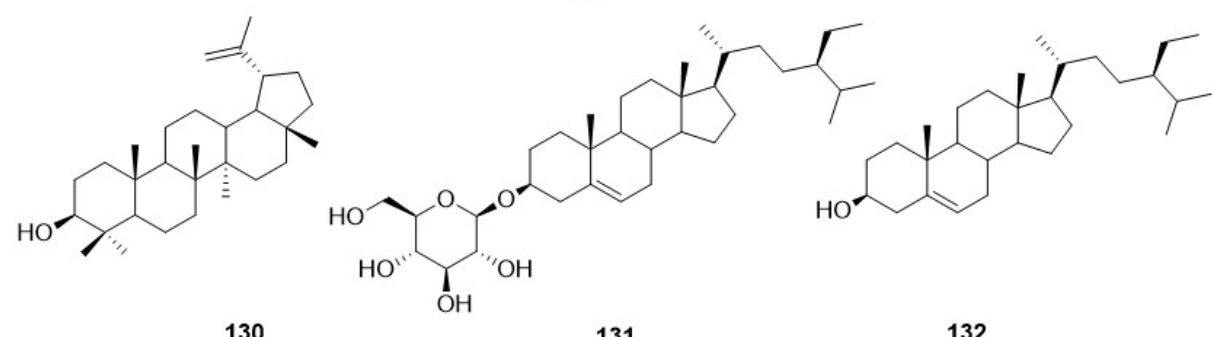

131

Figure 12. Other metabolites from Crepis taxa.

Table 5. Fixed oil composition of Crepis taxa.

\begin{tabular}{|c|c|c|c|c|}
\hline Taxa & Origin & Parts & Fatty Acids & Ref. \\
\hline C. alpina & USA & seeds & crepenynic acid; palmitic acid, stearic acid, oleic acid, linoleic acid & {$[115]$} \\
\hline C. aurea & Yugoslavia & seeds & $\begin{array}{l}\text { vernolic acid, crepenynic acid, palmitic acid, stearic acid, oleic acid, } \\
\text { linoleic acid }\end{array}$ & [116] \\
\hline C. biennis & Poland & seeds & $\begin{array}{l}\text { vernolic acid, crepenynic acid, palmitic acid, stearic acid, oleic acid, } \\
\text { linoleic acid }\end{array}$ & [116] \\
\hline C. conyzaefolia & USA & seeds & $\begin{array}{c}\text { (-)-S,S,-12-hydroxy-13-octadec-cis-9-enolide, } \\
\text { cis-12,13-epoxy-octadeca-trans-6-cis-9-dienoic acid, } \\
\text { cis-12,13-epoxyoctadeca-cis-6-cis-9-dienoic acid, vernolic acid }\end{array}$ & {$[117,118]$} \\
\hline C. foetida & Turkey & seeds & crepenynic acid, palmitic acid, stearic acid, oleic acid, linoleic acid & [116] \\
\hline $\begin{array}{l}\text { Crepis foetida ssp. } \\
\text { rhoeadifolia }\end{array}$ & Turkey & seeds & crepenynic acid, palmitic acid, stearic acid, oleic acid, linoleic acid & {$[116]$} \\
\hline C. intermedia & USA & seeds & $\begin{array}{c}\text { vernolic acid, crepenynic acid, palmitic acid, stearic acid, oleic acid, } \\
\text { linoleic acid }\end{array}$ & [116] \\
\hline C. lacera & Italy & $\begin{array}{l}\text { aerial } \\
\text { parts }\end{array}$ & $\begin{array}{c}\text { palmitoleic acid, stearic acid, palmitic acid, linolenic acid, linoleic acid, } \\
\text { oleic acid, isopalmitic acid }\end{array}$ & [111] \\
\hline C. occidentalis & USA & seeds & $\begin{array}{l}\text { vernolic acid, crepenynic acid, palmitic acid, stearic acid, oleic acid, } \\
\text { linoleic acid }\end{array}$ & [116] \\
\hline C. rubra & USA & seeds & crepenynic acid, palmitic acid, stearic acid, oleic acid, linoleic acid & [116] \\
\hline C. thomsonii & Pakistan & seeds & crepenynic acid, palmitic acid, stearic acid, oleic acid, linoleic acid & [116] \\
\hline \multirow{2}{*}{$\begin{array}{l}\text { C. vesicaria ssp. } \\
\text { taraxicifolia }\end{array}$} & Spain & seeds & $\begin{array}{l}\text { vernolic acid, crepenynic acid, palmitic acid, stearic acid, oleic acid, } \\
\text { linoleic acid }\end{array}$ & {$[116]$} \\
\hline & Portugal & leaves & $\begin{array}{c}\alpha \text {-linolenic acid, linoleic acid, oleic acid; palmitic acid, gondoic acid, } \\
\text { arachidic acid, stearic acid; margaric acid }\end{array}$ & [114] \\
\hline
\end{tabular}




\section{Essential Oils}

Despite the large number of species of genus Crepis, to the best of our knowledge, only one paper has been published on the chemical composition of their essential oils. It concerns the study of the volatile components of two odoriferous Crepis spp., namely C. rubra L. and C. foetida L., collected in Greece [119]. The volatile constituents of C. rubra flowers were characterised by the presence of sterols (38.9\%), fatty acids and esters (33.2\%), and hydrocabons (22.5\%), with $\beta$-sitosterol (38.9\%), eicosanoic acid (30.2\%) and heneicosane $(17.0 \%)$ being the main constituents. On the other hand, the stems-leaves volatiles revealed the absence of sterols and the occurrence of large amount of hydrocabons $(41.8 \%)$, followed by fatty acids and esters (33.2\%), and diterpenes $(8.6 \%)$. The main constituents of this oil were heneicosane $(11.4 \%)$, phytol $(8.6 \%)$, eicosanoic acid $(7.4 \%)$, salicylaldehyde $(6.4 \%)$, hexadecanol (5.7\%), and nonanal (5.0\%). The oil of the flowers of $C$. foetida L. was characterised by the presence hydrocabons (47.3\%) and phenylpropanoids $(40.0 \%)$, with salicylaldehyde $(40.0 \%)$, heneicosane $(18.2 \%)$, and octadecanol (15.9\%) being the main constituents of the oil. On the other hand, principal metabolites of the stems-leaves of C. foetida L. was carvacrol $(51.0 \%)$, although large amount of salicylaldehyde $(30.1 \%)$ was also detected.

\section{Biological Properties}

In the different subsections of Section 6, the different activities performed using different ssp. of Crepis genus are taken into consideration.

\subsection{Antibacterial and Antifungal Activity}

Antimicrobial resistance (AMR), which has sharply increased in recent years [120], is leading to an inability to treat different infectious diseases [121]. Bacteria, fungi, viruses, protozoa can show AMR towards the action of drugs. In fact, the massive use of antimicrobial prescriptions, and the excessive use of antibiotics in the agro-food system have contributed to the rapid increase in AMR. Well-founded studies have now shown that the annual deaths worldwide caused by AMR are equal to 750,000 units, and it is assumed that they will be more than 10 million by 2050 [122].

To counter this effect, secondary metabolites [123-125], produced by plant species as defence mechanisms, could represent a resource to be exploited for obtaining new drugs. Additionally, synthetic compounds [126-128] can be used to counteract the attack of parasites and pathogens before they can cause serious structural damage.

Ndom et al. [30] demonstrated that three lactone sesquiterpenes, $3 \beta, 9 \beta$-dihydroxyguaian4(15),10(14),11(13)-trien-6,12 olide (2), 3 $\beta, 8 \alpha$-dihydroxyguaian -4(15),10(14),11(13)-trien-6,12 olide (8-desacylcynaropicrin) (3), and $8 \alpha$-hydroxy- $4 \alpha(13), 11 \beta(15)$-tetrahydrozaluzanin C (47), chromatographically isolated from the aerial parts of $C$. camerronica, possess antibacterial activity against Staphylococcus aureus ATCC 13709, Escherichia coli ATCC 25922, and E. coli ATCC 35218 (inhibitory zone between 5 and $16 \mathrm{~mm}$ ), slightly lower than that of the streptomycin control (inhibitory zone between $2.5-6 \mathrm{~mm}$ ); however, none of the three compounds was found to be active against Pseudomonas aeruginosa ATCC 27853, Candida albicans ATCC 10231, and Klebsiella pneumoniae ATCC 10031.

The methanolic extract obtained from C. sancta leaves was evaluated against six strains of S. aureus isolated from soccer player's shoes in Balikesir Spor (U16 and U17) soccer team using the spread-plate method. The antibacterial results, obtained both as zone of inhibition, in $\mathrm{mm}$, and by broth dilution method, indicated that C. sancta extract showed a weak antibacterial activity only against the $S$. aureus BFT1 culture (inhibitory zone: $10 \mathrm{~mm}$; $\mathrm{MIC}=3250 \mu \mathrm{g} / \mathrm{mL}$ ). The antibiotics oxacillin, vancomycin, and erythromycin were used as positive controls, while methanol was used as negative control [129]. In a painstaking work [130], the antimicrobial activity of the extract obtained from the infusion of C. foetida $\mathrm{L}$. in boiling water was evaluated against four Gram-positive microorganisms (Streptococcus pneumoniae ATCC 19615, S. pyogenes ATCC 13615, S. aureus ATCC 25923, and S. epidermidis ATCC 12228), four Gram-negative bacteria (Klebsiella pneumoniae RSKK 574, Haemophilus influenzae ATCC 49766, P. aeruginosa ATCC 10145, and Acinetobacter baumannii RSKK 02026), 
and four fungal strains (C. albicans ATCC 10231, C. tropicalis, C. parapsilosis ATCC 22019, and C. krusei). The MIC value obtained against each microorganism was determined using broth microdilution techniques. The results showed a moderate activity of the aforementioned extract both towards Gram-negative and Gram-positive bacteria (MIC ranging from 32 to $64 \mu \mathrm{g} / \mathrm{mL}$ ), but excellent activity against the tested fungal strains (MIC values between 8-32 $\mu \mathrm{g} / \mathrm{mL})$. Interestingly, the extract (MIC $=32 \mu \mathrm{g} / \mathrm{mL}$ ) was 2 times more active than fluconazole control $(\mathrm{MIC}=64 \mu \mathrm{g} / \mathrm{mL})$ against the $C$. $k r u s e i$ strain.

\subsection{Antioxidant Activity}

The so-called reactive oxygen species (ROS), such as the superoxide anion $\left(\mathrm{O}_{2}{ }^{\bullet-}\right)$, and hydroxyl radical $\left(\mathrm{HO}^{\bullet}\right)$, are commonly produced by various biochemical reactions, and can produce, if present in high concentrations, serious damage to DNA, proteins, lipids, thus causing aging and cell death [131]. Scientific research has been addressed towards the study of new antioxidant compounds, obtained both from the natural source [132-136] and chemical-synthetic procedures [137], able to prevent and control the treatment of oxidative stress diseases, including cancer, cardiovascular and neurodegenerative diseases. It is also known that the antioxidant activity of a plant extract is highly correlated with the presence of phenolic compounds [138], and especially of phenolic acids [139]. Following phytochemical investigations, it emerged that species of the genus Crepis contain a good number of phenolic acids [61,140], inhibitors of oxidative processes. The HPLC-MS analysis of the aerial parts and roots of C. foetida L. subsp. rhoeadifolia showed the presence of chlorogenic acid (104) and luteonin (76). The tested extracts showed antioxidant activity in assays of DPPH $\left(\mathrm{IC}_{50}=0.26-1.54 \mathrm{mg} / \mathrm{mL}\right)$ and of the reactive substances of thiobarbituric acid (TBARS) (MDA level $=4.54-19.15 \mathrm{nmol} / \mathrm{mL}$ ), thus suggesting that the activity shown by the extracts of this plant is linked to its polyphenolic content [46]. In two other scientific works, the infusion, obtained in boiling water from the aerial parts of C. foetida L. [130], and the methanolic extract of C. sancta [129] were evaluated for their possible antioxidant capacity. None of the in vitro tests carried out with ABTS decolourisation assay and DPPH assay showed radical scavenging activity.

Finally, the hydro-alcoholic extract of the dehydrated leaves of C. vesicaria subsp. taraxacifolia, rich in L-chicoric acid (108) (130.5 mg per gram of plant), was evaluated for its antioxidant activity by DPPH, ABTS, and FRAP methods. The results showed that the extract had a good radical-scavenging activity and antioxidant action (DPPH $=\mathrm{IC}_{50}$ $26.20 \mu \mathrm{g} / \mathrm{mL}$; ABTS = IC $5018.92 \mu \mathrm{g} / \mathrm{mL} ;$ FRAP $=0.68 \mu \mathrm{g} / \mathrm{mL}$ of Trolox Equivalent) [61].

The absence of other assays besides the chemistry methods (DPPH, ABTS, FRAP) is a shortcoming, and further future studies in vivo would be desirable.

\subsection{Anti-Inflammatory Activity}

In the sole work conducted to evaluate the volatile content of Crepis species, the flowers, leaves, and stems of C. foetida and C. rubra [119] were investigated. As previously stated in Chapter 5, the main components of $C$. foetida were aromatic compounds such as salicylaldehyde (30.1-40.0\%) and carvacrol (51.0\%, present only in the stems), whereas the essential oils of C. rubra were characterised by the abundance of $\beta$-sitosterol (38.9\%, only in flowers), eicosanoic acid (7.4-30.2\%), and eneicosane (11.4-17.0\%). The fraction rich in salicylaldehyde from the essential oil of $C$. foetida flowers was studied for anti-inflammatory activity in vitro at different concentrations $(6.25,12.5,25.0,50.0 \mu \mathrm{g} / \mathrm{mL})$ in the HMEC-1 cell line for the reduction of ICAM-1 expression induced by TNF- $\alpha$. The activities highlighted a powerful reduction in ICAM-1 expression at $65-70 \%$ compared to the control. Further in vivo studies are needed to evaluate the potential of this essential oil.

In another paper, the ethanolic extract of $C$. vesciaria subsp. taraxacifolia leaves, rich in phenolic acids, namely derivatives of caffeic and ferulic acid as well as isomers of chicoric acid, was quantified by HPLC-PDA (high-performance chromatography with a diode array detector), showing inhibition of $\mathrm{NO}$ production in a dose-dependent manner with an $\mathrm{IC}_{50}$ of $0.43 \pm 0.01 \mathrm{mg} / \mathrm{mL}$ [61], confirming the use of this plant for inflammatory problems [46]. 


\subsection{Antidiabetic Activity}

Some Crepis species, due to the presence of numerous components, such as phenolic acids, steroids, and lignanoids, are used for the treatment of diabetes [43] and can significantly reduce blood glucose, increasing insulin resistance [114]. Li et al. [35] demonstrated, after having characterised the ethanolic extract obtained from C. crocea (Lam.) Babc., that the medium and high dose $(80 \mathrm{mg} / \mathrm{kg} / \mathrm{d}$ and $160 \mathrm{mg} / \mathrm{kg} / \mathrm{d}$, respectively) of three branched polysaccharides (CTP3-B, CTP3-C and CTP3-D) in KK-Ay mice resulted in a significant decrease in body weight or a reduction in the levels of blood glucose compared to the control group. This in vivo study suggests that regular use of polyacids can be employed in the treatment of type 2 diabetes and plays a key role in juggling glucose levels in the blood. Further studies should be conducted to evaluate the real mechanism of action of these acids and understand the hypoglycaemic effect.

\subsection{Antiulcer Activity}

Taraxinic acid-1'-O- $\beta$-D-glucopyranoside (72), a glycosylated sesquiterpene extracted from the roots of $C$. napifera, has been reported to have antiulcer action, inhibiting significantly $(p<0.01)$, at a dose of $70 \mathrm{mg} / \mathrm{kg}$, the development of gastric lesions induced in rats by aspirin, and not influencing the release of gastric secretion stimulated by histamine in the animal's stomach [83].

The methanolic extract of the widespread C. sancta [91], rich in 3-oxo- $\gamma$-costic acid (68), its methyl ester (69), and seven different methoxylated flavonoids (Table 3) was tested in vivo for a possible antiulcer action. At doses of $100 \mathrm{mg} / \mathrm{kg}$ and $200 \mathrm{mg} / \mathrm{kg}$, the extract tested on male albino mice showed an excellent antiulcer activity, comparable or even better than omeprazole, a typical drug used in antigastric activity. The histopathological examination showed a clear reduction in the infiltration of inflammatory cells, and the cessation of mucosal haemorrhage, with a dose-dependent reduction in the volume and titratable acidity of gastric juice which may explain the remarkable gastroprotective effect.

\subsection{Antiviral Activity}

Viruses represent a serious global, health, and even economic threat [141]. The past two years have taught us seriously what the explosion of new viruses entails. Scientific research has investigated possible antiviral agents starting from natural secondary structures. In a 2004 work, Ooi and collaborators [106] emphasised excellent antiviral activities of Youngia japonica (syn. Crepis japonica) ethanol extract against respiratory syncytial virus (RSV) in HEp-2 cells, capable of showing an inhibitory action equal to $50 \%\left(\mathrm{IC}_{50}\right)$ in doses between 3.0 and $6.0 \mathrm{~g} / \mathrm{mL}$, comparable values obtained from the action of the drug ribavirin. The selective index (SI), which is the ratio of the maximum non-cytotoxic concentration (MNCC) at the $\mathrm{IC}_{50}$ of the anti-RSV activity, was between 75 and 150, confirming the activity had taken over. The ethanolic extract, chromatographically focused, led to the isolation of three polyphenolic derivatives, 3,4-dicaffeylquinic acid (107), 3,5-dicaffeylquinic acid (106), and luteolin-7-O-glucoside (80). Both caffeic acid derivatives demonstrated strong antiviral activity against RSV in HEp-2 cells with an $\mathrm{IC}_{50}$ of $0.5 \mu \mathrm{g} / \mathrm{mL}$, while no anti-RSV activity was shown for the flavonoid [44]. Further structure-activity investigations are needed to understand the mechanism of action of caffeine derivatives.

\subsection{Cytotoxic Activity}

Eudesmane sesquiterpenes, 3-oxo-di-nor-eudesm-4-en-6 $\beta$-hydroxy-11-oic (66), 3-oxo$6 \beta$-hydroxy- $\gamma$-costic acid (67), 3-oxo- $\gamma$-costic acid (68), 3-oxo- $\gamma$-costic acid methyl ester (69), and (6R,9S)-roseoside (70), and two known methylated flavonoids acid from the acetone and the methanol extracts of $C$. sancta were subjected to an in vitro cytotoxicity assay against mouse lymphoma (L5178Y) cells. Only 3-oxo- $\gamma$-costic acid (68) and its methyl ester (69) demonstrated moderate cytotoxic activities ( $\mathrm{IC}_{50}$ of 21.0 and $9.5 \mu \mathrm{M}$ ), while the other three sesquiterpenes showed weak cytotoxic effects compared to kahalalide F, used as a control $\left(\mathrm{IC}_{50}=4.3 \mu \mathrm{M}\right)$. The antiproliferative activity of methyl ester was greater 
than that of the acid equivalent, with an $\mathrm{IC}_{50}$ value of $9.5 \mu \mathrm{M}$, and it can be attributed to increased lipophilicity of the methylene derivative favouring a better interaction with the target cellular receptor [90]. In another study, polar extracts obtained from Oriental hawksbeard [Youngia japonica (L.) DC.] were tested for potential anticancer activity in vitro against three cell lines: human promyelocytic leukemia (HL-60), human myeloid leukemia (chronic K-562), and mouse sarcoma 180 (S-180). The aqueous extract of Youngia japonica (syn. C. japonica) showed an inhibitory rate between 80 and $90 \%$ at the maximum dose of $450 \mathrm{~g} / \mathrm{mL}$ on the proliferation of HL-60 and K-562 cells, but significantly less activity in the suppression of S-180 cells (inhibition = 38\%) [106]. Not enough information can be gleaned from the work of Mikropoulou et al. [140]. In fact, only the decoction of C. sancta was cytotoxic at the dose tested against $\mathrm{C} 5 \mathrm{~N}$ and $\mathrm{A} 5$ using the MTT test.

Finally, the methanolic extract of C. foetida L. subsp. rhoeadifolia (Bieb.) Celak. inhibited the proliferation of HEPG-2, Caco-2, and MCF-7 cells with IC $_{50}$ values of $119.61,101.20$, and $90.94 \mathrm{~g} / \mathrm{mL}$, respectively. No inhibition was shown towards the proliferation of MCF-10A cells [104]. The major criticality is due to the fact that they are all tests carried out in vitro, using extracts in most of the experiments. There is a need to test the individual molecules, or all of them together, evaluating the synergistic effect and the mechanism of action.

\subsection{Toxicity}

Herbal medicines are often used to control various diseases. However, the presence of natural toxins and pollutants can cause severe adverse reactions, such as severe liver damage [142]. For example, in some plants belonging to the Asteraceae family, such as Doronicum orientale Hoffm., there are toxins such as pyrrolizidine alkaloids which, if ingested incorrectly or in large quantities, can cause biochemical reactions with serious damage to the liver [143]. In this context, the traditional use of C. rueppellii Sch. Beep. in the treatment of hepatic syndromes, including jaundice and hepatitis, was confirmed by a preliminary study by Fleurentin et al. [49]. The ethanolic extract of C. rueppellii at a dose of $200 \mathrm{mg} / \mathrm{kg}$ showed hepatoprotective properties against hepatotoxicity in mice induced by injection of specific doses of ethanol and carbon tetrachloride, significantly reducing the mortality of the treated subjects $(p<0.05)$ and the significant increase in plasma GPT activity $(p<0.01)$. The lower and higher doses $(100 \mathrm{mg} / \mathrm{kg}$ and $400 \mathrm{mg} / \mathrm{kg}$, respectively) did not show hepatoprotective effects. The anti-necrotic action, however, must be demonstrated and evaluated through biochemical studies trying to correlate it with any specific active principles.

Three sesquiterpene lactones, 8-epi-desacylcynaropicrin-3-O- $\beta$-glucopyranoside (10), 8-epigrosheimin (24), and 8- $\beta$-hydroxidehydrozaluzanine $C(5)$, isolated from the aerial parts of $C$. lacera, were examined by in vitro and in vivo studies to evaluate their relative toxicity. Indeed, several farmers in southern Italy have complained of the death of grazing ruminants, such as sheep and goats, after the ingestion of large quantities of C. lacera. Cell viability was evaluated in cell cultures of the Madin Darby Bovine Kidney (MDBK) bovine kidney cell line after incubation with active molecules. The three sesquiterpenes were shown to be cytotoxic after $48 \mathrm{~h}$ of incubation, and in vivo studies on adult male Sprague-Dawley rats showed significant lesions $(p<0.05)$ in the liver, lungs and kidneys when treated with $2 \mathrm{mg} / \mathrm{kg}$ of C. lacera polar extract [47]. In-depth studies need to be conducted to understand the mechanism of action of the secondary metabolites present in this plant.

\section{Conclusions}

Agricultural landscapes have changed substantially in recent decades, from the dominance of smallholder fields with wide diversity to large-scale monoculture systems where ecosystem services are much more challenged. These species, with annual or biennial behaviour, especially when in relationship and balance with other species, make a substantial contribution to the development of resilient patterns that respond to the principles of agroecology and enhance pathways of ecological transition. 
In this context, Crepis L. has attracted great interest in many Mediterranean environments. In fact, since ancient times, the Crepis genus has been used in the agri-food field. Up to January 2022, 132 metabolites have been isolated from the roots and aerial parts of the different species, identified using spectroscopic techniques, and evaluated for biological potential.

Among these, the guaianic-type sesquiterpenes and the glycosylated and non-glycosylated methylated flavonoids are the most characteristic. In particular, phytochemical studies have shown the presence of typical sesquiterpene structures: the costus lactone type, by far the most represented, with fifty-two compounds, hypocretenolides, and the lactucin type. Especially in the last two decades, the extracts obtained from the different parts of the plants and the pure isolated compounds have been tested and evaluated for their biological activities, such as antitumor, anti-inflammatory, antiviral, antimicrobial, antiulcer, antioxidant, and nutritional. However, the few studies on biological properties of Crepis species present in literature do not make it possible to confirm and validate the edibility of this genus or its use, in the form of a decoction, in traditional medicine.

Nevertheless, major critical issues remain. Most scientific studies have aimed at testing the extracts or compounds isolated through in vitro studies; biological support obtained in vivo and biochemical investigations relating to the mechanism of action of the tested samples are lacking. Only one work reports the isolation of essential oils, which could be used for a chemotaxonomic distinction between dubious species.

This literature review aims to direct current researchers to work on a widely promising genus at the agri-food level, given the massive incidence of numerous biologically exploitable sesquiterpene compounds, due to the different structural variety.

Supplementary Materials: The following supporting information can be downloaded at: https: / / www.mdpi.com/article/10.3390/plants11040519/s1. Table S1. Crepis taxa studied so far and their synonymous. Accepted botanical name in bold according to The Plant List [2].

Author Contributions: Conceptualisation and methodology, N.B., F.S. and M.B.; writing-original draft preparation, N.B., F.S. and M.B.; writing-review and editing, N.B., F.S. and M.B. All authors have read and agreed to the published version of the manuscript.

Funding: This work was supported by the Ministero dello Sviluppo Economico (Italy)—Project title: "Innovazioni tecnologiche bio-based e potenziamento dell'economia circolare nella gestione degli scarti da lavorazione primaria di mandorle biologiche con elevate potenzialità agroindustriali" \#F/200037/01-03/X45. This work was supported by grant from MIUR-ITALY PRIN 2017 (Project No. 2017A95NCJ).

Institutional Review Board Statement: Not applicable.

Informed Consent Statement: Not applicable.

Conflicts of Interest: The authors declare no conflict of interest.

\section{References}

1. Kilian, N.; Gemeinholzer, B.; Lack, H.W. Cichorieae. In Systematics Evolution, and Biogeography of Compositae; Funk, V.A., Susanna, A., Stuessy, T.F., Bayer, R.J., Eds.; International Association for Plant Taxonomy: Bratislava, Slovakia, 2009; pp. 343-383.

2. Bremer, K. Asteraceae, Cladistics and Classification; Timber Press: Portland, OR, USA, 1994.

3. The Plant List. Available online: http://www.theplantlist.org/ (accessed on 10 January 2022).

4. Babcock, E.B. The Genus Crepis. Part One: The Taxonomy, Phylogeny, Distribution and Evolution of Crepis; University of California Press: Oakland, CA, USA, 1947.

5. Enke, N.; Gemeinholzer, B. Babcock revisited: New insights into generic delimitation and character evolution in Crepis L. (Compositae: Cichorieae) from ITS and MatK sequence data. Taxon 2008, 57, 756-768. [CrossRef]

6. Guerra, J.G.; Cabello, F.; Fernández-Quintanilla, C.; Peña, J.M.; Dorado, J. How weed management influence plant community composition, taxonomic diversity and crop yield: A long-term study in a Mediterranean vineyard. Agric. Ecosyst. Environ. 2022, 326, 107816. [CrossRef]

7. Dudley, N.; Alexander, S. Agriculture and biodiversity: A review. Biodiversity 2017, 18, 45-49. [CrossRef]

8. Tilman, D.; Fargione, J.; Wolff, B.; D’Antonio, C.; Dobson, A.; Howarth, R.; Schindler, D.; Schlesinger, W.H.; Simberloff, D.; Swackhamer, D. Forecasting agriculturally driven global environmental change. Science 2001, 292, 281. [CrossRef] [PubMed] 
9. Ollerton, J.; Winfree, R.; Tarrant, S. How many flowering plants are pollinated by animals. Oikos 2011, 120, 321-326. [CrossRef]

10. Travlos, I.S. Weeds in Perennial Crops as an unexpected tool of integrated crop management. In Weeds and Their Ecological Functions; Taab, A., Ed.; Nova Publishers: New York, NY, USA, 2013; pp. 97-113.

11. Juárez-Escario, A.; Conesa, J.A.; Solé-Senan, X.O. Management as a driver of functional patterns and alien species prominence in weed communities of irrigated orchards in Mediterranean areas. Agric. Ecosyst. Environ. 2017, 249, 247-255. [CrossRef]

12. Mas, M.T.; Poggio, S.L.; Verdú, A.M.C. Weed community structure of mandarin orchards under conventional and integrated management in northern Spain. Agric. Ecosyst. Environ. 2007, 119, 305-310. [CrossRef]

13. Fried, G.; Cordeau, S.; Metay, A.; Kazakou, E. Relative importance of environmental factors and farming practices in shaping weed communities structure and composition in French vineyards. Agric. Ecosyst. Environ. 2019, 275, 1-13. [CrossRef]

14. Radicetti, E.; Mancinelli, R.; Campiglia, E. Impact of managing cover crop residues on the floristic composition and species diversity of the weed community of pepper crop (Capsicum annuum L.). Crop Prot. 2013, 44, 109-119. [CrossRef]

15. Ganser, D.; Knop, E.; Albrecht, M. Sown wildflower strips as overwintering habitat for arthropods: Effective measure or ecological trap? Agric. Ecosyst. Environ. 2019, 275, 123-131. [CrossRef]

16. Alcalá Herrera, R.; Ruano, F.; Gálvez Ramírez, C. Attraction of green lacewings (Neuroptera: Chrysopidae) to native plants used as ground cover in woody Mediterranean agroecosystems. Biol. Control 2019, 139, 104066. [CrossRef]

17. Sikorska, D.; Macegoniuk, S.; Łaszkiewicz, E.; Sikorski, P. Energy crops in urban parks as a promising alternative to traditional lawns-Perceptions and a cost-benefit analysis. Urban For. Urban Green. 2020, 49, 126579. [CrossRef]

18. Russo, A.; Bruno, M.; Avola, R.; Cardile, V.; Rigano, D. Reduction of endogenous defense as a possible mechanism by which chamazulene-rich Artemisia arborescens essential oils affect the cell growth of human melanoma cells. Plants 2020, 9, 1000. [CrossRef] [PubMed]

19. Ammar, S.; Noui, H.; Djamel, S.; Madani, S.; Maggi, F.; Bruno, M.; Romano, D.; Canale, A.; Pavela, R.; Benelli, G. Essential oils from three Algerian medicinal plants (Artemisia campestris, Pulicaria arabica and Saccocalyx satureioides) as new botanical insecticides? Environ. Sci. Pollut. Res. 2020, 27, 26594-26604. [CrossRef] [PubMed]

20. Bruno, M.; Modica, A.; Catinella, G.; Canlı, C.; Arasoglu, T.; Çelik, S. Chemical composition of the essential oil of Centaurea tomentella Hand.-Mazz. and C. haussknechtii Boiss. (Asteraceae) collected wild in Turkey and its activity on microorganisms affecting historical art craft. Nat. Prod. Res. 2019, 33, 1092-1100. [CrossRef]

21. Bruno, M.; Bancheva, S.; Maggio, A.; Rosselli, S. Sesquiterpenoids in subtribe Centaureinae (Cass.) Dumort (tribe Cardueae, Asteraceae): Distribution, ${ }^{13} \mathrm{C}-\mathrm{NMR}$ spectral data and biological properties. Phytochemistry 2013, 95, 19-93. [CrossRef]

22. Formisano, C.; Rigano, D.; Senatore, F.; Bancheva, S.; Maggio, A.; Rosselli, S.; Bruno, M. Flavonoids in Subtribe Centaureinae (Cass.) Dumort (tribe Cardueae, Asteraceae): Distribution and ${ }^{13} \mathrm{C}-\mathrm{NMR}$ spectral data. Chem. Biodiv. 2012, 9, $2096-2158$. [CrossRef]

23. Formisano, C.; Rigano, D.; Russo, A.; Cardile, V.; Caggia, S.; Arnold, N.A.; Mari, A.; Piacente, S.; Rosselli, S.; Senatore, F.; et al. Phytochemical profile and apoptotic activity of Onopordum cynarocephalum. Planta Med. 2012, 78, 1651-1660. [CrossRef]

24. Bruno, M.; Maggio, A.; Rosselli, S.; Safder, M.; Bancheva, S. The metabolites of the genus Onopordum (Asteraceae): Chemistry and biological properties. Curr. Org. Chem. 2011, 15, 888-927. [CrossRef]

25. Page, M.J.; McKenzie, J.E.; Bossuyt, P.M.; Boutron, I.; Hoffmann, T.C.; Mulrow, C.D.; Shamseer, L.; Tetzlaff, J.M.; Akl, E.A.; Brennan, S.E.; et al. The PRISMA 2020 Statement: An Updated Guideline for Reporting Systematic Reviews. Int. J. Surg. 2021, 88, 105906. [CrossRef]

26. Idolo, M.; Motti, R.; Mazzoleni, S. Ethnobotanical and phytomedicinal knowledge in a long-history protected area, the Abruzzo, Lazio and Molise National Park (Italian Apennines). J. Ethnopharmacol. 2010, 127, 379-395. [CrossRef] [PubMed]

27. Schicchi, R.; Geraci, A. Verdure Spontanee per l'Alimentazione e La Salute; Palermo University Press: Palermo, Italy, 2021.

28. De Natale, A.; Menale, B.; Di Lecce, S.; Pollio, A. An ethnobotanical survey of wild edible plants of Campania (Italy). Bull. Reg. Nat. Hist. 2021, 1, 45-110. [CrossRef]

29. Lentini, F.; Venza, F. Wild food plants of popular use in Sicily. J. Ethnobiol. Ethnomed. 2007, 3, 15. [CrossRef] [PubMed]

30. Ndom, J.C.; Mbafor, J.T.; Wansi, J.D.; Kamdem, A.W.; Meva'a, L.M.; Vardamides, J.C.; Toukam, F.; Pegyemb, D.; Ngando, T.M.; Laatsch, H.; et al. Sesquiterpene lactones from Crepis camerronica (Asteraceae). Nat. Prod. Res. 2006, 20, 435-442. [CrossRef]

31. Uncini Manganelli, R.E.; Camangi, F.; Tomei, P.E. L'uso delle Erbe nella Tradizione Rurale della Toscana; Agenzia Regionale per lo Sviluppo e 1'Innovazione nel Settore Agricolo-Forestale: Florence, Italy, 2007; Volume 1, p. 92.

32. Schröder, E. Ethnobotanik-Ethnobotany: Beiträge und Nachträge zur 5. Internationalen Frachkonferenz; Springer: Berlin/Heidelberg, Germany, 1980; p. 347.

33. Vardavas, C.I.; Majchrzak, D.; Wagner, K.H.; Elmadfa, I.; Kafatos, A. Lipid concentrations of wild edible greens in Crete. Food Chem. 2006, 99, 822-834. [CrossRef]

34. Zeghichi, S.; Kallithraka, S.; Simopoulos, A.P.; Kypriotakis, Z. Nutritional composition of selected wild plants in the diet of Crete. In Plants in Human Health and Nutrition Policy; Karger: Basel, Switzerland, 2003; Volume 91, pp. 22-40.

35. Li, Y.; Li, S.; He, S.; Yue, Y.; Ni, Y. Structural analysis and antidiabetic activity study of three acidic-type polysaccharides from Crepis crocea (Lam.) Babc. Nat. Prod. Res. 2021, 35, 4988-4993. [CrossRef]

36. Singh, K.N.; Lal, B. Ethnomedicines used against four common ailments by the tribal communities of Lahaul-Spiti in western Himalaya. J. Ethnopharmacol. 2008, 115, 147-159. [CrossRef] 
37. Singh, K.N. Traditional knowledge on ethnobotanical uses of plant biodiversity: A detailed study from the Indian western Himalaya. Biodiv. Res. Conserv. 2012, 28, 63-77. [CrossRef]

38. Altundag, E.; Ozturk, M. Ethnomedicinal studies on the plant resources of east Anatolia, Turkey. Procedia Soc. Behav. Sci. 2011, 19, 756-777. [CrossRef]

39. Çakılcıŏlu, U.; Sengün, M.T.; Türkoğlu, Đ. An ethnobotanical survey of medicinal plants of Yazıkonak and Yurtbası districts of Elazı̆̆ province, Turkey. J. Med. Plants Res. 2010, 4, 567-572. [CrossRef]

40. Ertuğ, F. An ethnobotanical study in central Anatolia (Turkey). Econ. Bot. 2000, 54, 155-182. [CrossRef]

41. Kisiel, W.; Barszcz, B. Phenolics from Crepis rhoeadifolia. Fitoterapia 1996, 67, 284-285.

42. Chamberlin, R.V. The ethno-botany of the Gosiute Indians of Utah. In Memoirs of the American Anthropological Association; Leopold Classic Library: Amsterdam, The Netherlands, 1911; Volume 5, Part 5.

43. Dalar, A. Plant taxa used in the treatment of diabetes in Van Province, Turkey. Int. J. Second. Metab. 2018, 5, 170-184. [CrossRef]

44. Munira, S.; Kabir, H.; Bulbul, I.J.; Nesa, L.; Muhit, A.; Haque, I. Pharmacological activities of Youngia japonica extracts. Annu. Res. Rev. Biol. 2018, 25, 1-14. [CrossRef]

45. Ooi, L.S.; Wang, H.; He, Z.; Ooi, V.E. Antiviral activities of purified compounds from Youngia japonica (L.) DC (Asteraceae, Compositae). J. Ethnopharmacol. 2006, 106, 187-191. [CrossRef] [PubMed]

46. Rahman, A.H.M.M. An ethnobotanical investigation on Asteraceae family at Rajshahi, Bangladesh. Acad. J. Med. Plants 2013, 1 , 92-100. [CrossRef]

47. Russo, R.; Restucci, B.; Vassalo, A.; Cortese, L.; D’Ambola, M.; Montagnaro, S.; Ciarcia, R.; Florio, S.; De Tommasi, N.; Severino, L. Toxicity of Crepis lacera in grazing ruminants. BMC Vet. Res. 2018, 14, 74. [CrossRef]

48. Khatib, C.; Nattouf, A.; Hasan Agha, M.I. Ethnobotanical survey of medicinal herbs in the Western region in Syria (Latakia and Tartus). Res. Sq. 2021, 1-75. [CrossRef]

49. Fleurentin, J.; Hoefler, C.; Lexas, A.; Mortierb, F.; Pelt, J.M. Hepatoprotective properties of Crepis rueppellii and Anisotes trisulcus: Two traditional medicinal plants of Yemen. J. Ethnopharmacol. 1986, 16, 105-111. [CrossRef]

50. Chekole, G.; Asfaw, Z.; Kelbessa, E. Ethnobotanical study of medicinal plants in the environs of Tara-gedam and Amba remnant forests of Libo Kemkem District, northwest Ethiopia. J. Ethnobiol. Ethnomed. 2015, 11, 4. [CrossRef]

51. Akyol, Y.; Altan, Y. Ethnobotanical studies in the Maldan Village (Province Manisa, Turkey). Marmara Pharm. J. 2013, 17, 21-25. [CrossRef]

52. Kilic, O.; Bagci, E. An ethnobotanical survey of some medicinal plants in Keban (Elazı̆̆-Turkey). J. Med. Plants Res. 2013, 7 , 1675-1684. [CrossRef]

53. Sansanelli, S.; Tassoni, A. Wild food plants traditionally consumed in the area of Bologna (Emilia Romagna region, Italy). J. Ethnobiol. Ethnomed. 2014, 10, 69. [CrossRef] [PubMed]

54. Ranfa, A.; Bodesmo, M. An ethnobotanical investigation of traditional knowledge and uses of edible wild plants in the Umbria Region, Central Italy. J. Appl. Bot. Food Qual. 2017, 90, 246-258. [CrossRef]

55. Biscotti, N.; Del Viscio, G.; Bonsanto, D. Indagine etnobotanica sull'uso alimentare tradizionale di piante selvatiche in un comprensorio montano della regione Puglia (Subappennino Dauno, Provincia di Foggia). Atti Soc. Tosc. Sci. Nat. Mem. Ser. B 2018, 125, 17-29. [CrossRef]

56. Pieroni, A.; Nebel, S.; Cassandra Quave, C.; Münz, H.; Heinrich, M. Ethnopharmacology of liakra: Traditional weedy vegetables of the Arbëreshë of the Vulture area in southern Italy. J. Ethnopharmacol. 2002, 81, 165-185. [CrossRef]

57. Atzei, A.D. Le Piante nella Tradizione Popolare della Sardegna; Delfino, C., Ed.; Carlo Delfino Publisher \& C. Snc: Sassari, Italy, 2003

58. Loi, M.C.; Poli, F.; Sacchetti, G.; Selenu, M.B.; Ballero, M. Ethnopharmacology of Ogliastra (Villagrande Strisaili, Sardinia, Italy). Fitoterapia 2004, 75, 277-295. [CrossRef]

59. Gonzalez-Tejero, M.R.; Molero-Mesa, J.; Casares-Porcel, M.; Martinez Lirola, M.J. New contributions to the ethnopharmacology of Spain. J. Ethnopharmacol. 1995, 45, 157-165. [CrossRef]

60. Simopoulos, A.P.; Gopalan, C. (Eds.) Plants in Human Health and Nutrition Policy; Karger Medical and Scientific Publishers: Basel, Switzerland, 2003; Volume 91, pp. 22-40. [CrossRef]

61. Harborne, J.B. The rare flavone isoetin as a yellow flower pigment in Heywoodiella oligocephala and in other Cichorieae. Phytochemistry 1978, 17, 915-917. [CrossRef]

62. Panfili, G.; Niro, S.; Bufano, A.; D’Agostino, A.; Fratianni, A.; Paura, B.; Falasca, L.; Cinquanta, L. Bioactive compounds in wild asteraceae edible plants consumed in the mediterranean diet. Plant Foods Hum. Nutr. 2020, 75, 540-546. [CrossRef]

63. Zidorn, C. Sesquiterpene lactones and their precursors as chemosystematic markers in the tribe Cichorieae of the Asteraceae. Phytochemistry 2008, 69, 2270-2296. [CrossRef]

64. Ahmed, A.A.; Mahmoud, A.A.; Hammam, M.A.S.; Abd El-Razek, M.H.; Mabry, T.J. A sesquiterpene lactone and furocoumarin from Crepis aspera. Rev. Latinoam. Quim. 2000, 28, 143-145.

65. Kisiel, W. Hypocretenolides from Crepis aurea. Fitoterapia 1994, 65, 381.

66. Michalska, K.; Kisiel, W.; Zidorn, C. Sesquiterpene lactones from Crepis aurea (Asteraceae, Cichorieae). Biochem. System. Ecol. 2013, 46, 1-3. [CrossRef]

67. Kisiel, W.; Kohlmünzer, S. Ixerin F from Crepis biennis. Planta Med. 1987, 53, 390. [CrossRef] [PubMed]

68. Kisiel, W. Sesquiterpene lactone glycosides from Crepis capillaris. Phytochemistry 1984, 23, 1955-1958. [CrossRef]

69. Kisiel, W. 8-Epidesacylcynaropicrin from Crepis capillaris. Planta Med. 1983, 49, 246-247. [CrossRef] 
70. Kisiel, W. Two new guaianolides from Crepis capillaris. Pol. J. Chem. 1983, 57, 139-143.

71. Kotti, I.; Barda, C.; Karioti, A.; Skaltsa, H. Sesquiterpene lactones and other secondary metabolites from Crepis commutata (Spreng.) Greuter-Asteraceae. Biochem. System. Ecol. 2019, 86, 103917. [CrossRef]

72. Kisiel, W.; Michalska, K. Sesquiterpenoids and phenolics from Crepis conyzifolia. Zeitschrift für Naturforschung C 2001, 56, 961-964. [CrossRef]

73. Kisiel, W.; Jakupovic, J.; Huneck, S. Guaianolides from Crepis crocea. Phytochemistry 1994, 35, 269-270. [CrossRef]

74. Tsoukalas, M.; Gousiadou, C.; Skaltsa, H. Guaianolides and phenolic constituents from Crepis dioscoridis L., growing wild in Greece. Phytochem. Lett. 2014, 7, 202-206. [CrossRef]

75. Kisiel, W.; Barszcz, B. Sesquiterpenoids and phenolics from roots of Crepis foetida. Pol. J. Chem. 1999, 73, 569-571.

76. Barda, C.; Ciric, A.; Soković, M.; Tsoukalas, M.; Skaltsa, H. Phytochemical investigation of Crepis incana Sm. (Asteraceae) endemic to southern Greece. Biochem. System. Ecol. 2018, 80, 59-62. [CrossRef]

77. Miyase, T.; Ueno, A.; Noro, T.; Kuroyanagi, M.; Fukushima, S. Studies on sesquiterpene glycosides from Crepis japonica Benth. Chem. Pharm. Bull. 1985, 33, 4451-4456. [CrossRef]

78. Giambanelli, E.; D’Antuono, L.F.; Ferioli, F.; Frenich, A.G.; Romero-González, R. Sesquiterpene lactones and inositol 4hydroxyphenylacetic acid derivatives in wild edible leafy vegetables from Central Italy. J. Food Comp. Anal. 2018, 72, 1-6. [CrossRef]

79. Kassem, F.F. Guaianolides from Crepis micrantha growing in Egypt. Alex. J. Pharm. Sci. 2007, 21, 55-58.

80. Kisiel, W.; Zielińska, K.; Joshi, S.P. Sesquiterpenoids and phenolics from Crepis mollis. Phytochemistry 2000, 54, 763-766. [CrossRef]

81. Fazylova, A.S.; Turdybekov, K.M.; Kadirberlina, G.M.; Rakhimova, B.B.; Adekenov, S.M. Molecular structure of crepidioside A and isolipidiol from Crepis multicaulis. Chem. Nat. Compd. 2000, 36, 177-180. [CrossRef]

82. Zhao, A.; Peng, X.; Tang, C.; Zhang, R. Isolation and identification of sesquiterpenes from Crepis napifera. Yaoxue Xuebao 2000, 35, 442-444.

83. Wu, S.; Luo, X.; Ma, Y.; Hao, X.; Wu, D. Anti-gastric ulcer sesquiterpene glycosides from Crepis napifera. Yaoxue Xuebao 2002, 37, 33-36.

84. Michalska, K.; Szneler, E.; Kisiel, W. Structure elucidation of an unusual new sesquiterpene lactone glucoside ester from Crepis pannonica. Magn. Res. Chem. 2016, 54, 175-177. [CrossRef] [PubMed]

85. Kisiel, W.; Gromek, D. Guaianolides from Crepis pulchra. Pol. J. Chem. 1994, 68, 535-538. [CrossRef]

86. Casinovi, C.G.; Fardella, G.; Rossi, C. 4,5-Didydro-11-nor-11-hydroxy-D7,11-santonin, a new nor-sesquiterpene- $\gamma$-lactone found in Crepis pygmaea. Planta Med. 1982, 44, 186-187. [CrossRef] [PubMed]

87. Rossi, C.; Evidente, A.; Menghini, A. A nor-sesquiterpene-c-lactone found in Crepis pygmaea. Phytochemistry 1985, $24,603-604$. [CrossRef]

88. Kisiel, W.; Barszcz, B. Sesquiterpene lactone glycosides from Crepis pyrenaica. Phytochemistry 1995, 39, 1395-1397. [CrossRef]

89. Kisiel, W.; Barszcz, B. Sesquiterpene lactone lactones from Crepis rhoeadifolia. Phytochemistry 1996, 43, 823-825. [CrossRef]

90. Ebada, S.S.; El-Kashef, D.H.; Müller, W.E.G.; Proksch, P. Cytotoxic eudesmane sesquiterpenes from Crepis sancta. Phytochem. Lett. 2019, 33, 46-48. [CrossRef]

91. Ebada, S.S.; Al-Jawabri, N.A.; Youssef, F.S.; Albohy, A.; Aldalaien, S.M.; Disi, A.M.; Proksch, P. In vivo antiulcer activity, phytochemical exploration, and molecular modelling of the polyphenolic-rich fraction of Crepis sancta extract. Inflammopharmacology 2020, 28, 321-331. [CrossRef]

92. Kisiel, W.; Kohlmünzer, S. Sesquiterpene lactones from Crepis setosa Hall. Acta Soc. Bot. Pol. 1990, 59, 81-85. [CrossRef]

93. Kisiel, W. Guaianolides from Crepis sibirica. Fitoterapia 1995, 66, 276.

94. Kisiel, W.; Kohlmünzer, S. Sesquiterpene lactone glycosides from Crepis tectorum. Pol. J. Chem. 1989, 63, 527-530. [CrossRef]

95. Kisiel, W.; Kohlmünzer, S. A sesquiterpene lactone glycoside from Crepis tectorum. Phytochemistry 1989, 28, 2403-2404. [CrossRef]

96. Adekenov, S.M.; Kadirberlina, G.M.; Turdybekov, K.M.; Struchkov, Y.T. Terpenoids of Crepis tectorum. Molecular and crystal structure of the sesquiterpene lactone 8-epideacylcinaropicrin. Chem. Nat. Compd. 1991, 27, 562-565. [CrossRef]

97. Zidorn, C.; Ellmerer-Müller, E.P.; Stuppner, H. Guaianolides from Calycocorsus stipitatus and Crepis tingitana. Phytochemistry 1999, 50, 1061-1062. [CrossRef]

98. Barbetti, P.; Casinovi, C.G.; Santurbano, B.; Longo, R. A grosheimin from Crepis virens. Collect. Czech. Chem. Commun. 1979, 44, 3123-3127.

99. Kisiel, W.; Michalska, K.; Szneler, E. Sesquiterpene lactones from Crepis zacintha. Pol. J. Chem. 2002, 76, 1571-1576. [CrossRef]

100. Zidorn, C.; Schubert, B.; Stuppner, H. Phenolics as chemosystematic markers in and for the genus Crepis (Asteraceae, Cichorieae). Sci. Pharm. 2008, 76, 743-750. [CrossRef]

101. Màñez, S.; Recio, M.C.; Giner, R.M.; Sanz, M.J.; Terencio, M.C.; Peris, J.B.; Stübing, G.; Rios, J.L. A chematoxonomic review of the subtribe crepidinase based on its phenol constituents. Biochem. System. Ecol. 1994, 22, 297-305. [CrossRef]

102. Zidorn, C.; Schubert, B.; Stuppner, H. Altitudinal differences in the contents of phenolics in flowering heads of three members of the tribe Lactuceae (Asteraceae) occurring as introduced species in New Zealand. Biochem. Syst. Ecol. 2005, 33, 855-872. [CrossRef]

103. Rees, S.; Harborne, J.B. Flavonoids and other phenolics of Cichorium and related members of the Lactuceae (Compositae). Bot. J. Linn. Soc. 1984, 89, 313-319. [CrossRef] 
104. Zengin, G. Crepis foetida L. subsp. rhoeadifolia (Bieb.) Celak. as a source of multifunctional agents: Cytotoxic and phytochemical evaluation. J. Funct. Foods 2015, 17, 698-708. [CrossRef]

105. Bakar, F.; Acıkara, Ö.B.; Ergene, B.; Nebioğlu, S.; Çitoğlu, G.S. Antioxidant activity and phytochemical screening of some asteraceae plants. Turk. J. Pharm. Sci. 2015, 12, 123-132. [CrossRef]

106. Ooi, L.S.; Wang, H.; Luk, C.-W.; Ooi, V.E. Anticancer and antiviral activities of Youngia japonica (L.) DC (Asteraceae, Compositae). J. Ethnopharmacol. 2004, 94, 117-122. [CrossRef]

107. Mànez, S.; Giner, R.M.; Recio, M.C.; Terencio, M.C.; Rios, J.L. Phenolics of Crepis and their taxonomic implications. Planta Med. 1992, 58 (Suppl. S1), A698-A699. [CrossRef]

108. Zidorn, C.; Stuppner, H. Evaluation of chemosystematic characters in the genus Leontodon. Taxon 2001, 50, 115-133. [CrossRef]

109. Fraisse, D.; Felgines, C.; Texier, O.; Lamaison, J.L. Caffeoyl derivatives: Major antioxidant compounds of some wild herbs of the Asteraceae family. Food Nutr. Sci. 2011, 2, 181-192. [CrossRef]

110. Zidorn, C.; Ellmerer-Müller, E.P.; Stuppner, H. A germacranolide and three hydroxybenzyl alcohol derivatives from Hieracium murorum and Crepis bocconi. Phytochem. Anal. 2001, 12, 281-285. [CrossRef]

111. Epifano, F.; Genovese, S. Euphorbol acetate from Crepis lacera. Chem. Nat. Comp. 2012, 48, 910-911. [CrossRef]

112. He, S.; Li, Y.; Li, S.; Peng, Z.; Li, X.; Ni, Y. Structural characterization of the polysaccharide CTP3-E from Crepis turczaniowii C. A Mey. Chin. Pharm. J. 2013, 48, 1959-1964. [CrossRef]

113. Ni, Y.; Lu, F.; Hao, X. Study on chemical constituents in Crepis turczanilwii. Chin. J. Inf. Tradit. Chin. Med. 2007, 14, 51-52.

114. Pedreiro, S.; da Ressurreição, S.; Lopes, M.; Cruz, M.T.; Batista, T.; Figueirinha, A.; Ramos, F. Crepis vesicaria L. subsp. taraxacifolia leaves: Nutritional profile, phenolic composition and biological properties. Int. J. Environ. Res. Public Health 2021, 18, 151. [CrossRef] [PubMed]

115. Neff, W.E.; Adlof, R.O.; Konishi, H.; Weisleder, D. High-performance liquid chromatography of the triacylglycerols of Vernonia galamensis and Crepis alpina seed oils. J. Am. Oil Chem. Soc. 1993, 70, 449-455. [CrossRef]

116. Earle, F.R.; Barclay, A.S.; Wolff, I.A. Compositional variation in seed oils of the Crepis genus. Lipids 1966, 1, 325-327. [CrossRef] [PubMed]

117. Spencer, G.F.; Plattner, R.D.; Wayne Miller, R. (-)-(S,S)-12-hydroxy-13-octadec-cis-9-enolide, a 14-membered lactone from Crepis conyzaefolia seed oil. Phytochemistry 1977, 16, 764-766. [CrossRef]

118. Spencer, G.F. Epoxyoctadecadienoic acids from Crepis conyzaefolia seed oil. Phytochemistry 1977, 16, 282-284. [CrossRef]

119. Barda, C.; Grafakou, M.E.; Kalpoutzakis, E.; Heilmann, J.; Skaltsa, H. Chemical composition of Crepis foetida L. and C. rubra L. volatile constituents and evaluation of the in vitro anti-inflammatory activity of salicylaldehyde rich volatile fraction. Biochem. System. Ecol. 2021, 96, 104256. [CrossRef]

120. Badalamenti, N.; Ilardi, V.; Rosselli, S.; Bruno, M. The ethnobotany, phytochemistry and biological properties of genus Ferulago-A review. J. Ethnopharmacol. 2021, 274, 114050. [CrossRef]

121. Mcewen, S.A.; Collignon, P.J. Antimicrobial resistance: A one health perspective. Microbiol. Spectr. 2018, 6, 2. [CrossRef]

122. O'Neill, J. Tackling Drug-Resistant Infections Globally: Final Report and Recommendations; Government of the United Kingdom: London, UK, 2016.

123. Badalamenti, N.; Russi, S.; Bruno, M.; Maresca, V.; Vaglica, A.; Ilardi, V.; Zanfardino, A.; Di Napoli, M.; Varcamonti, M.; Cianciullo, P.; et al. Dihydrophenanthrenes from a sicilian accession of Himantoglossum robertianum (Loisel.) P. Delforge showed antioxidant, antimicrobial, and antiproliferative activities. Plants 2021, 10, 2776. [CrossRef]

124. Di Napoli, M.; Maresca, V.; Varcamonti, M.; Bruno, M.; Badalamenti, N.; Basile, A.; Zanfardino, A. (+)-(E)-Chrysanthenyl acetate: A molecule with interesting biological properties contained in the Anthemis secundiramea (Asteraceae) flowers. Appl. Sci. 2020, 10, 6808. [CrossRef]

125. Bruno, M.; Milia, A.; Catinella, G.; Bancheva, S. Volatile components from aerial parts of Centaurea diffusa and C. micrantha ssp. melanosticta and their biocidal activity on microorganisms affecting historical art crafts. Nat. Prod. Commun. 2018, 13, 1179-1182 [CrossRef]

126. Mattio, L.; Catinella, G.; Iriti, M.; Vallone, L. Inhibitory activity of stilbenes against filamentous fungi. Ital. J. Food Saf. 2021, 10, 8461. [CrossRef] [PubMed]

127. Mattio, L.M.; Catinella, G.; Dallavalle, S.; Pinto, A. Stilbenoids: A natural arsenal against bacterial pathogens. Antibiotics 2020, 9, 336. [CrossRef] [PubMed]

128. Catinella, G.; Mattio, L.M.; Musso, L.; Arioli, S.; Mora, D.; Beretta, G.L.; Zaffaroni, N.; Pinto, A.; Dallavalle, S. Structural requirements of benzofuran derivatives dehydro- $\delta$-and dehydro- $\varepsilon$-viniferin for antimicrobial activity against the foodborne pathogen listeria monocytogenes. Int. J. Mol. Sci. 2020, 21, 2168. [CrossRef]

129. Okmen, A.S. Antioxidant and antibacterial activities of different plants extracts against Staphylococcus aureus isolated from soccer player's shoes and knowledge and applications about foot hygiene of the soccer players. Afr. J. Tradit. Complement. Altern. Med. 2015, 12, 143. [CrossRef]

130. Didem, D.O.; Berrin, O.; Sanem, H.; Mecit, V. Assessment of antioxidant, antibacterial, antimycobacterial, and antifungal activities of some plants used as folk remedies in Turkey against dermatophytes and yeast-like fungi. Turk. J. Biol. 2012, 36, 672-686. [CrossRef]

131. Badalamenti, N.; Modica, A.; Ilardi, V.; Bruno, M. Chemical constituents and biological properties of genus Doronicum (Asteraceae). Chem. Biodivers. 2021, 18, e2100631. [CrossRef] 
132. Badalamenti, N.; Modica, A.; Ilardi, V.; Bruno, M.; Maresca, V.; Zanfardino, A.; Di Napoli, M.; Castagliuolo, G.; Varcamonti, M.; Basile, A. Daucus carota subsp. maximus (Desf.) Ball from Pantelleria, Sicily (Italy): Isolation of essential oils and evaluation of their bioactivity. Nat. Prod. Res. 2021, in press. [CrossRef]

133. Badalamenti, N.; Ilardi, V.; Rosselli, S.; Bruno, M.; Maggi, F.; Leporini, M.; Falco, T.; Loizzo, M.R.; Tundis, R. Ferulago nodosa subsp. geniculata (Guss.) Troia \& Raimondo from Sicily (Italy): Isolation of essential oil and evaluation of its bioactivity. Molecules 2020, 25, 3249. [CrossRef]

134. Rosselli, S.; Tundis, R.; Bruno, M.; Leporini, M.; Falco, T.; Gagliano Candela, R.; Badalamenti, N.; Loizzo, M.R. Ceiba speciosa (A. St.-Hil.) seeds oil: Fatty acids profiling by GC-MS and NMR and bioactivity. Molecules 2020, 25, 1037. [CrossRef] [PubMed]

135. Sut, S.; Maggi, F.; Bruno, S.; Badalamenti, N.; Quassinti, L.; Bramucci, M.; Beghelli, D.; Lupidi, G.; Dall'Acqua, S. Hairy Garlic (Allium subhirsutum) from Sicily (Italy): LC-DAD-MSn analysis of secondary metabolites and in vitro biological properties. Molecules 2020, 25, 2837. [CrossRef] [PubMed]

136. Magurano, F.; Sucameli, M.; Picone, P.; Micucci, M.; Baggeri, M.; Marchi, A.; Bucci, P.; Gioacchini, S.; Catinella, G.; Borgonovo, G.; et al. Antioxidant activity of Citrus limonoids and investigation of their virucidal potential against SARS-CoV-2 in cellular models. Antioxidants 2021, 10, 1794. [CrossRef] [PubMed]

137. Cardullo, N.; Catinella, G.; Floresta, G.; Muccilli, V.; Rosselli, S.; Rescifina, A.; Bruno, M.; Tringali, C. Synthesis of rosmarinic acid amides as antioxidative and hypoglycemic agents. J. Nat. Prod. 2019, 82, 573-582. [CrossRef]

138. Tawaha, K.; Alali, F.Q.; Gharaibeh, M.; Mohammad, M.; El-Elimat, T. Antioxidant activity and total phenolic content of selected Jordanian plant species. Food Chem. 2007, 104, 1372-1378. [CrossRef]

139. Sytar, O. Phenolic acids in the inflorescences of different varieties of buckwheat and their antioxidant activity. J. King Saud. Univ. Sci. 2015, 27, 136-142. [CrossRef]

140. Mikropoulou, E.; Vougogiannopoulou, K.; Kalpoutzakis, E.; Sklirou, A.; Skaperda, Z.; Houriet, J.; Wolfender, J.L.; Trougakos, I.; Kouretas, D.; Halabalaki, M.; et al. Phytochemical composition of the decoctions of greek edible greens (chórta) and evaluation of antioxidant and cytotoxic properties. Molecules 2018, 23, 1541. [CrossRef]

141. Mattio, L.M.; Catinella, G.; Pinto, A.; Dallavalle, S. Natural and nature-inspired stilbenoids as antiviral agents. Eur. J. Med. Chem. 2020, 202, 112541. [CrossRef]

142. Quan, N.V.; Dang Xuan, T.; Teschke, R. Potential hepatotoxins found in herbal medicinal products: A systematic review. Int. J. Mol. Sci. 2020, 21, 5011. [CrossRef]

143. Teschke, R.; Vongdala, N.; Quan, N.V.; Quy, T.N.; Dang Xuan, T. Metabolic toxification of 1,2-unsaturated pyrrolizidine alkaloids causes human hepatic sinusoidal obstruction syndrome: The updateint. Int. J. Mol. Sci. 2021, 22, 10419. [CrossRef] 